TRANSACTIONS OF THE

AMERICAN MATHEMATICAL SOCIETY

Volume 356, Number 8, Pages 3301-3323

S 0002-9947(03)03381-6

Article electronically published on November 12, 2003

\title{
THE COHOMOLOGY OF CERTAIN HOPF ALGEBRAS ASSOCIATED WITH $p$-GROUPS
}

\author{
JUSTIN M. MAUGER
}

\begin{abstract}
We study the cohomology $H^{*}(A)=\operatorname{Ext}_{A}^{*}(k, k)$ of a locally finite, connected, cocommutative Hopf algebra $A$ over $k=\mathbb{F}_{p}$. Specifically, we are interested in those algebras $A$ for which $H^{*}(A)$ is generated as an algebra by $H^{1}(A)$ and $H^{2}(A)$. We shall call such algebras semi-Koszul. Given a central extension of Hopf algebras $F \rightarrow A \rightarrow B$ with $F$ monogenic and $B$ semiKoszul, we use the Cartan-Eilenberg spectral sequence and algebraic Steenrod operations to determine conditions for $A$ to be semi-Koszul. Special attention is given to the case in which $A$ is the restricted universal enveloping algebra of the Lie algebra obtained from the mod- $p$ lower central series of a $p$-group. We show that the algebras arising in this way from extensions by $\mathbb{Z} /(p)$ of an abelian $p$-group are semi-Koszul. Explicit calculations are carried out for algebras arising from rank $2 p$-groups, and it is shown that these are all semiKoszul for $p \geq 5$.
\end{abstract}

\section{INTRODUCTION}

A quadratic algebra over a field $k$ is an associative algebra of the form $A=$ $T(V) /(R)$, where $(R)$ is the two-sided ideal of relations generated by some $R \subset$ $V \otimes V$, and $V$ is a locally finite graded vector space. In [13, Priddy defines a quadratic algebra $A$ to be a Koszul algebra if $H^{*}(A)=\operatorname{Ext}_{A}^{*}(k, k)$ is generated as an algebra by $\operatorname{Ext}_{A}^{1}(k, k)$. Basic examples include tensor, polynomial, and exterior algebras.

When $A$ is Koszul, Priddy constructs projective resolutions over $A$ which are "small" subcomplexes of the bar resolution. He also shows that if $H^{*}(A)$ is itself Koszul, then $A \simeq H^{*}\left(H^{*}(A)\right)$ (Koszul duality). Equivalent definitions of Koszulity include:

(1) A certain complex associated to A, the Koszul complex, is acyclic [10] Theorem 1.2].

(2) There exists a graded projective resolution

$$
\cdots \rightarrow P^{2} \rightarrow P^{1} \rightarrow P^{0} \rightarrow k \rightarrow 0
$$

of $k$ such that $P^{i}$ is generated by its component of degree $i, P^{i}=A \cdot P_{i}^{i}$ [2] Definition 1.2.1]. Equivalently, $\operatorname{Ext}_{A}^{i, j}(k, k)=0$ if $i \neq j$.

Now assume $A=T(V) /(R)$ is a more general algebra which is not necessarily quadratic, i.e. $R \subset \sum_{i \geq 1} V^{\otimes i}$. We are interested in the case when $H^{*}(A)$ is

Received by the editors April 30, 2002 and, in revised form, April 2, 2003.

2000 Mathematics Subject Classification. Primary 16E40; Secondary 16S37, 16S30.

Key words and phrases. Koszul algebras, cohomology of algebras, Hopf algebras. 
generated as an algebra by $H^{1}(A)$ and $H^{2}(A)$. Following Priddy, we shall call such algebras semi-Koszul. The quintessential example is the truncated polynomial algebra $\mathbb{F}_{p}[x] /\left(x^{p}\right)$, whose cohomology is generated by a one-dimensional exterior class $z$ and a two-dimensional polynomial class $u$. In this example, $u=-\widetilde{\beta \mathcal{P}}^{0}(z)$. In general, it will often be the case that the generators from $H^{2}$ are related to those of $H^{1}$ by some algebraic Bockstein operation or Massey product. Since we allow $A$ to not be quadratic, none of the results cited above for Koszul algebras necessarily hold for semi-Koszul algebras. It is unknown at this time whether there are any interesting relationships between $A$ and $H^{*}\left(H^{*} A\right)$, or anything like a semi-Koszul resolution.

We will focus on locally finite, connected, cocommutative Hopf algebras over $k=$ $\mathbb{F}_{p}$. Consequently, we will be able to use algebraic Steenrod operations. In Section 3 we analyze extensions of Hopf algebras $F \rightarrow A \rightarrow B$, where $F=\mathbb{F}_{p}[x] /\left(x^{p}\right)$ is central in $A$. There is a first quadrant spectral sequence converging to $H^{*}(A)$ with $E_{2}^{* *} \simeq H^{*}(B) \otimes H^{*}(F)$. The behavior of this spectral sequence is determined by the differential $d_{2}(1 \otimes z)=\mu \in H^{2}(B)$, where $z \in H^{1}(F)$. We prove in Theorem 3.3 that $A$ is semi-Koszul provided that $B$ is semi-Koszul, $\widetilde{\beta \mathcal{P}}^{0}(\mu)=0$ and the annihilator ideal of $\mu$ is either zero or generated in dimension one.

Section 4 delves into Hopf algebras arising from $p$-groups. For a finite $p$-group $G$, the mod- $p$ lower central series filtration $\left\{\Gamma^{i} G\right\}$ has as associated graded object a restricted Lie algebra $\mathcal{L} G$. The universal restricted enveloping algebra $\mathcal{V} \mathcal{L} G$ is isomorphic to the associated graded algebra $E^{0}\left(\mathbb{F}_{p} G\right)$ of the group ring $\mathbb{F}_{p} G$ with respect to the augmentation ideal filtration [15. Working with Lie algebras is helpful because many of the algebraic Steenrod operations vanish there, in particular $\widetilde{\mathcal{P}}^{0}$ 14. In Section 4.2 we explore central extensions of $p$-groups $H \rightarrow G \rightarrow Q$, where $H \simeq \mathbb{Z} /(p)$. Using Theorem 3.3. we show that $\mathcal{V} \mathcal{L} G$ is semi-Koszul if $Q$ is abelian (Theorem 4.15). In Section 4.3, we prove a corresponding statement for Hopf algebras which do not necessarily arise from $p$-groups. Given a central extension of Hopf algebras $F \rightarrow A \rightarrow B$ with $F=\mathbb{F}_{p}[x] /\left(x^{p}\right)$, we show that $E^{0} A$ is semi-Koszul provided that $B$ is commutative.

A rank two $p$-group is a finite $p$-group whose largest elementary abelian subgroup has rank two. In Section 5 we show that $\mathcal{V} \mathcal{L} G$ is semi-Koszul for all rank two $p$ groups $G$ with $p \geq 5$. One would hope to show that this holds for any finite $p$-group, but such is not the case. A counterexample is given in 5.3 .1

Section 2 introduces notation and covers the necessary algebraic preliminaries such as algebraic Steenrod operations and the Cartan-Eilenberg spectral sequence associated with a central extension of algebras. We relegate to the Appendix definitions of the cobar complex $C^{*}(A)$, Massey products in $H^{*}(A)$ and computations in the cohomology of $\mathbb{F}_{p}[x] /\left(x^{p^{n}}\right)$.

\section{Algebraic PReliminaries}

In this section we introduce notation and conventions. For more details on the cohomology of algebras, the reader is referred to Adams [1, Chapter 2]. We also summarize algebraic Steenrod operations, the Cartan-Eilenberg spectral sequence, and Lie algebras.

Let $k=\mathbb{F}_{p}$ be the field of $p$ elements. By an algebra $A$ we mean a non-negatively graded, associative $k$-algebra with product $\mu: A \otimes A \rightarrow A$, unit $\eta: k \rightarrow A$ 
and augmentation $\epsilon: A \rightarrow k$. We assume $A$ is locally finite, that is, each $A_{n}$ is finite dimensional over $k$. We also assume $A$ is connected, i.e. $A_{0}=k$. Let $I=I(A)=\operatorname{ker} \epsilon$ denote the augmentation ideal.

Tensor products are taken over $k$, unless otherwise noted. The tensor product of two algebras $A$ and $B$ is defined by $(A \otimes B)_{n}=\sum_{i+j=n} A_{i} \otimes B_{j}$, with multiplication $(a \otimes b)(x \otimes y)=(-1)^{\operatorname{deg} b \operatorname{deg} x} a x \otimes b y$. If the algebras in question are bigraded, we define $(A \otimes B)_{n, m}=\sum_{\substack{i+s=n \\ j+t=m}} A_{i, j} \otimes B_{s, t}$, and the total degree is used in the multiplication formula. For $x \in A_{s, t}, x$ has homological degree (or dimension) $s$, internal degree $t$ and total degree $s+t$. By the commutativity of $A$ we shall mean graded-commutativity, also called anticommutativity: $a b=(-1)^{\operatorname{deg} a \operatorname{deg} b} b a$.

A Hopf algebra is an algebra with coproduct, or diagonal, $\psi: A \rightarrow A \otimes A$ which is an algebra map. We shall assume that $\psi$ is (graded) coassociative and cocommutative. Since the algebra $A$ is assumed to be connected, it has an antipode $\sigma: A \longrightarrow A$, but we shall not make use of it. See [12 for more details on Hopf algebras and their structure.

Let $\operatorname{Ext}_{A}^{i}(-, k)$ denote the $i$-th right derived functor of $\operatorname{Hom}_{A}(-, k)$ in the category of graded left $A$-modules. The cohomology $H^{*}(A)$ of $A$ is defined to be $\operatorname{Ext}_{A}^{*}(k, k)$, where $k$ is given an $A$-module structure by the augmentation map $\epsilon$. The Yoneda product makes this a graded algebra [1]. We recall some basic facts about the cohomology of algebras.

(1) Let $A, B$ be locally finite algebras. Then $H^{*}(A \otimes B) \simeq H^{*}(A) \otimes H^{*}(B)$.

(2) If $A$ is a connected cocommutative Hopf algebra with unit, then $H^{*}(A)$ is anticommutative.

We introduce the following definitions.

\section{Definition 2.1.}

(1) A graded algebra $C$ is called bigenerated if it is generated as an algebra by elements of homological degree 1 and 2.

(2) An algebra $A$ is semi-Koszul if $C=H^{*}(A)$ is bigenerated.

We shall make use of a reindexed form of algebraic Steenrod operations. In 11. Theorem 11.8], May defines operations $\mathcal{P}^{i}: H^{s, t}(A) \rightarrow H^{s+(2 i-t)(p-1), p t}(A)$ for $p \geq 3$. These operations are zero if $2 i<t$ or $2 i>s+t$. We would like to reindex these so that $\mathcal{P}^{i}$ raises homological degree by $2 i(p-1)$ and is trivial for $i<0,2 i>s$. (May denotes these reindexed operations by $\widetilde{\mathcal{P}}^{i}$.) This regrading has the effect of making $\widetilde{\mathcal{P}}^{0}$ the first possibly non-trivial operation on $H^{*}(A)$. It also eliminates all operations on $H^{s, t}(A)$ for $p>2$ when $t$ is odd. This will not be of concern to us, as most of our algebras will be concentrated in even degrees.

Theorem 2.2. Let $A$ be a cocommutative Hopf algebra over $\mathbb{F}_{p}$. There exist natural homomorphisms

(1) $\widetilde{S q}^{i}: H^{s, t}(A) \longrightarrow H^{s+i, 2 t}(A)$ for $p=2$,

(2) $\widetilde{\mathcal{P}}^{i}: H^{s, 2 t}(A) \longrightarrow H^{s+2 i(p-1), 2 p t}(A)$ and

$\widetilde{\beta \mathcal{P}}^{i}: H^{s, 2 t}(A) \longrightarrow H^{s+2 i(p-1)+1,2 p t}(A)$ for $p \geq 3$

with the following properties. 
(1) $\widetilde{S q}^{i}=0$ if $i<0$ or $i>s$,

$\widetilde{\mathcal{P}}^{i}=0$ if $i<0$ or $2 i>s$,

$\widetilde{\beta \mathcal{P}}^{i}=0$ if $i<0$ or $2 i \geq s ;$

(2) $\widetilde{S q}^{i}(x)=x^{2}$ if $i=s$,

$\widetilde{\mathcal{P}}^{i}(x)=x^{p}$ if $2 i=s$.

(3) Cartan formulae

$\widetilde{S q}^{i}(x y)=\sum_{j=0}^{i} \widetilde{S q}^{j}(x) \widetilde{S q}^{i-j}(y)$,

$\widetilde{\mathcal{P}}^{i}(x y)=\sum_{j=0}^{i} \widetilde{\mathcal{P}}^{j}(x) \widetilde{\mathcal{P}}^{i-j}(y)$,

$\widetilde{\beta \mathcal{P}}^{i}(x y)=\sum_{j=0}^{i}\left(\widetilde{\beta \mathcal{P}}^{j}(x) \widetilde{\mathcal{P}}^{i-j}(y)+\widetilde{\mathcal{P}}^{j}(x) \widetilde{\beta \mathcal{P}}^{i-j}(y)\right) ;$

(4) Adem relations

(a) if $a<2 b$,

$$
\widetilde{S q} \widetilde{S q}^{b}=\sum_{i}\left(\begin{array}{c}
b-i-1 \\
a-2 i
\end{array}\right) \widetilde{S q}^{a+b-i} \widetilde{S q}^{i}
$$

(b) if $a<p b$,

$$
{\widetilde{\beta^{\epsilon \mathcal{P}}}}^{a} \widetilde{\mathcal{P}}^{b}=\sum_{i}(-1)^{a+i}\left(\begin{array}{c}
(b-i)(p-1)-1 \\
a-p i
\end{array}\right) \widetilde{\beta}^{\epsilon \mathcal{P}}{ }^{a+b-i} \widetilde{\mathcal{P}}^{i}
$$

(c) if $a \leq p b$,

$$
\begin{aligned}
\widetilde{\beta \mathcal{P P}} \widetilde{\mathcal{P}}^{b}= & (1-\epsilon) \sum_{i}(-1)^{a+i}\left(\begin{array}{c}
(b-i)(p-1)-1 \\
a-p i
\end{array}\right) \widetilde{\beta \mathcal{P}}^{a+b-i} \widetilde{\mathcal{P}}^{i} \\
& -\sum_{i}(-1)^{a+i}\left(\begin{array}{c}
(b-i)(p-1)-1 \\
a-p i-1
\end{array}\right) \widetilde{\beta \mathcal{P}}^{a+b-i} \widetilde{\beta \mathcal{P}}^{i}
\end{aligned}
$$

where $\epsilon=0$ or 1 , the binomial coefficients are taken modulo $p$ and, by abuse of notation, $\widetilde{\beta^{0 \mathcal{P}}} s=\widetilde{\mathcal{P}}^{s}$ and $\widetilde{\beta^{1 \mathcal{P}}} s=\widetilde{\beta \mathcal{P}}^{s}$.

Remark. One can actually define operations $\widetilde{\mathcal{P}}^{i / 2}, \widetilde{\beta \mathcal{P}}^{i / 2}$ on elements of odd internal degree such that the Cartan formulae and Adem relations still make sense. See 16. Appendix 1.5] for details.

A decreasing filtration of $A$ is a sequence of $k$-modules $A=F^{0} A \supset F^{1} A \supset$ $F^{2} A \supset \cdots$ such that $F^{s} A \cdot F^{t} A \subset F^{s+t} A$. Let $\rho_{s}$ denote the natural map $F^{s} A \rightarrow$ $F^{s / s+1} A=F^{s} A / F^{s+1} A$. We will sometimes suppress the $A$ when it is clear from context. We will say that elements in $F^{s+i}$ are of lower filtration level than elements in $F^{s}$, even though the indices are greater. Let the filtration degree of an element $x \in A$ be the largest $i$ such that $x \in F^{i} A$ (if such an $i$ exists).

Let $F \subset A$ be a subalgebra. We say $F$ is normal in $A$ if $I(F) \cdot A=A \cdot I(F)$. In this case, there is a well-defined quotient $B=A / A \cdot I(F)$ denoted by $A / / F$. In the case that $F$ is a sub- Hopf algebra, $B$ is also a Hopf algebra. We say $F$ is central in $A$ if each element of $F$ anticommutes with each element of $A$. By an extension or exact sequence $F \rightarrow A \rightarrow B$ of algebras we shall mean that $F$ is normal in $A$ and $B \simeq A / / F$.

Theorem 2.3. Let $F \stackrel{i}{\rightarrow} A \stackrel{\pi}{\rightarrow} B$ be a central extension of graded, connected algebras. There is a first quadrant spectral sequence converging to $H^{*}(A)$ with

(1) $E_{2}^{s, t} \simeq H^{s}(B) \otimes H^{t}(F)$.

(2) $E_{\infty}^{s, t} \simeq F^{s} H^{s+t}(A) / F^{s+1} H^{s+t}(A)$ where $\left\{F^{s}\right\}$ is a decreasing filtration of $H^{*}(A)$.

(3) The ring structure of $H^{s}(B) \otimes H^{t}(F)$ is defined by $(x \otimes y)(z \otimes w)=(-1)^{\left(t+t^{\prime}\right)\left(s+s^{\prime}\right)} x z \otimes y w$, where $y \in H^{t, t^{\prime}}(F), z \in H^{s, s^{\prime}}(B)$. 
(4) $d_{r}: E_{r}^{s, t} \rightarrow E_{r}^{s+r, t-r+1}$ is a graded derivation, meaning $d_{r}(a b)=d_{r}(a) \cdot b+(-1)^{\operatorname{deg} a} a \cdot d_{r}(b)$.

(5) The map $H^{s}(B) \simeq E_{2}^{s, 0} \rightarrow E_{\infty}^{s, 0} \simeq F^{s} H^{s}(A) \longrightarrow H^{s}(A)$ corresponds to the induced $\operatorname{map} \pi^{*}: H^{*}(B) \rightarrow H^{*}(A)$.

The map $H^{t}(A)=F^{0} H^{t}(A) \simeq E_{\infty}^{0, t} \longmapsto E_{2}^{0, t} \simeq H^{t}(F)$ corresponds to the induced map $i^{*}: H^{*}(A) \rightarrow H^{*}(F)$.

(6) Kudo transgression theorem. Assume, in addition, that $F \stackrel{i}{\rightarrow} A \stackrel{\pi}{\rightarrow} B$ is an extension of Hopf algebras over $\mathbb{F}_{p}$. The transgression $d_{r}: E_{r}^{0, r-1} \rightarrow E_{r}^{r, 0}$ anticommutes with Steenrod operations: if $x \in E_{r}^{0, r-1}$ and $\theta$ is a Steenrod operation of homological degree $i$, then $\theta(x)$ survives until $E_{r+i}^{0, r+i-1}$ and $d_{r+i}(\theta(x))=(-1)^{i} \theta\left(d_{r}(x)\right)$.

Since the algebras $F, A$ and $B$ are graded, the spectral sequence is actually trigraded, with the third grading being the internal degree. We have suppressed this third grading, as it is preserved by the differentials $d_{r}$. This spectral sequence was used by Adams [1 2.3.1]. Ravenel [16, A1.3.14] calls it the Cartan-Eilenberg spectral sequence. For (6) see [17, Proposition 2.3].

The Lie algebras we shall work with are all graded over either $\mathbb{Z}$ or $\mathbb{F}_{p}$. A restricted Lie algebra is a Lie algebra over $\mathbb{F}_{p}$ with restriction $\xi$.

For $L$ a restricted Lie algebra, let $\operatorname{Tens}(L)$ be the tensor algebra on $L$, and let $J \subset \operatorname{Tens}(L)$ be the two-sided ideal generated by elements of the form $x \otimes y-$ $(-1)^{\operatorname{deg} x \operatorname{deg} y} y \otimes x-[x, y]$ and $x^{\otimes p}-\xi(x)$ for $x, y \in L$.

Definition 2.4. The universal restricted enveloping algebra of $L$ is defined to be $\mathcal{V} L=\operatorname{Tens}(L) / J$.

We can put a Hopf algebra structure on $\mathcal{V} L$ by setting $\psi(x)=x \otimes 1+1 \otimes x$, $\epsilon(x)=0$ for $x \in L$. The cohomology of a Lie algebra $L$ is defined to be $H^{*}(\mathcal{V} L)$.

Theorem 2.5 ([14, 5.3], [11, Theorem 8.5]). Let $L$ be a Lie algebra over $\mathbb{F}_{p}$. Then the Steenrod operations $\widetilde{\mathcal{P}}^{0}$ and $\widetilde{S_{q}}$ are identically zero on $H^{*}(\mathcal{V} L)$.

\section{An extension theorem}

Let $A$ be a cocommutative Hopf algebra over $\mathbb{F}_{p}$. In this section, we will find sufficient conditions for $H^{*}(A)$ to be bigenerated. Let $z \in A$ be a central primitive element of height $p$. This means $z$ anticommutes with every element of $A, z^{p}=0$ and $z^{i} \neq 0$ if $i<p$. Let $F=\mathbb{F}_{p}[z] /\left(z^{p}\right)$ be the central sub-Hopf algebra of $A$ generated by $z$. Let $B=A / / F$. We have the central extension of Hopf algebras

$$
F \longrightarrow A \longrightarrow B
$$

with corresponding spectral sequence

$$
E_{2}^{s, t} \simeq H^{s}(B) \otimes H^{t}(F) \Rightarrow H^{s+t}(A)
$$

Let $n$ be the internal degree of $z \in F$. Note that when $p$ is odd, $n$ must be even. We recall that the cohomology of the fiber $F$ is

$$
\begin{aligned}
& H^{*}(F) \simeq \Lambda[\zeta] \otimes \mathbb{F}_{p}[\epsilon], \quad \zeta \in H^{1, n}, \epsilon \in H^{2, n p}, \quad \text { with } \widetilde{\beta \mathcal{P}}^{0}(\zeta)=-\epsilon \quad p \text { odd }, \\
& H^{*}(F) \simeq \mathbb{F}_{2}[\zeta], \quad \zeta \in H^{1, n}, \quad \text { with } \widetilde{S q}^{1}(\zeta)=\zeta^{2} \quad p=2 .
\end{aligned}
$$

See Theorem A.9 for more details on the Steenrod operations. 
Theorem 3.3 (Main Theorem). Let $F, A, B$ be as in (3.1). In the spectral sequence (3.2), let $d_{2}(\zeta)=\mu \in H^{2, n}(B)$. Assume

(1) $\widetilde{\beta \mathcal{P}}^{0}(\mu)=0$ or $\widetilde{S q}^{1} \mu=0$.

(2) The annihilator ideal of $\mu$ in $H^{*}(B)$ is either zero or is generated in dimension one.

(3) B is semi-Koszul.

Then $A$ is semi-Koszul.

Proof. By the transgression theorem 2.3(6),

$$
\begin{aligned}
d_{3}(\epsilon) & =-\widetilde{\beta \mathcal{P}}^{0}(\mu), \quad p \text { odd }, \\
d_{3}\left(\zeta^{2}\right) & =\widetilde{S q}^{1}(\mu), \quad p=2 .
\end{aligned}
$$

If $\widetilde{\beta \mathcal{P}}^{0}(\mu)=0$ or $\widetilde{S q}^{1}(\mu)=0$, then

$$
\begin{aligned}
& d_{2 p+1}\left(\epsilon^{p}\right)=\widetilde{\mathcal{P}}^{1}\left(d_{3}(\epsilon)\right)=0 \quad \text { or } \\
& d_{5}\left(\zeta^{4}\right)=\widetilde{S q}^{2}\left(d_{3}\left(\zeta^{2}\right)\right)=0 .
\end{aligned}
$$

Likewise for all other powers of $\epsilon$ or $\zeta$. Thus in this case, the spectral sequence (3.2) collapses at $E_{3}$.

In order for $H^{*}(A)$ to be bigenerated, we need $E_{\infty}$ to be bigenerated. Assume $\widetilde{\beta \mathcal{P}}^{0}(\mu)=0$ or $\widetilde{S q}^{1}(\mu)=0$. If $\mu$ is a zero divisor in $H^{*}(B)$, we may run into the following problem. Let $\eta \in H^{i}(B)$ be in the annihilator ideal of $\mu$. Then

$$
d_{2}(\eta \otimes \zeta)=d_{2}(\eta) \otimes \zeta+(-1)^{i} \eta \otimes d_{2}(\zeta)= \pm \eta \otimes \mu=0 .
$$

Nothing can hit $\eta \otimes \zeta \in E_{r}^{i, 1}$ because $d_{r} \equiv 0$ for $r \geq 3$, and $d_{2} \equiv 0$ from the $E_{2}^{*, 2}$ line. Thus $\eta \otimes \zeta$ is a non-bounding permanent cycle of dimension $i+1$. If $i=1$, then $\eta \otimes \zeta \in E_{\infty}^{1,1}$ is a two-dimensional generator of $E_{\infty}$. If $i>1$ on the other hand, $\eta \otimes \zeta$ is a possibly indecomposable element in $E_{\infty}^{i, 1}$ of homological dimension larger than 2. (It will be indecomposable if, for example, $\eta$ is indecomposable in $H^{*}(B)$.) Consequently $E_{\infty}$ - and thus $H^{*}(A)$-is not bigenerated.

Assume then that the annihilator ideal of $\mu$ in $H^{*}(B)$ is either zero or is generated multiplicatively by elements of dimension one. Assume also that $H^{*}(B)$ is bigenerated. Then $E_{\infty}$ has the following possible set $S$ of multiplicative generators:

(1) $1 \otimes \epsilon$ for $p$ odd, $1 \otimes \zeta^{2}$ for $p=2$;

(2) elements of the form $\eta \otimes \zeta$, where $\eta \in H^{1}(B)$ and $\eta \mu=0$;

(3) elements $\sigma \otimes 1, \nu \otimes 1$, where $\sigma \in H^{1}(B)$ and $\nu \in H^{2}(B)$;

(4) $1 \otimes \zeta$ (if $d_{2} \equiv 0$ ).

All these elements have homological degree less than or equal to two, and thus $E_{\infty}$ is bigenerated. Recall that $H^{*}(A)$ has a decreasing filtration $F^{*} H^{*}(A)$, and that we denote the natural projection $F^{s} H^{n}(A) \rightarrow F^{s / s+1} H^{n}(A) \simeq E_{\infty}^{s, n-s}$ by $\rho_{s}$. Choose a set of representatives $T \subset H^{*}(A)$ for each of the types of generators in $S$ :

(1) $e \in F^{0} H^{2}(A)$;

(2) $x \in F^{1} H^{2}(A)$;

(3) $\alpha \in F^{1} H^{1}(A), v \in F^{2} H^{2}(A)$;

(4) $z \in F^{0} H^{1}(A)$. 
We claim that $H^{*}(A)$ is generated as an algebra by $T$, from which it follows that $A$ is semi-Koszul. Let $S^{\prime}=S \cup\{1 \otimes 1\}, T^{\prime}=T \cup\{1\}$. The proof will proceed by reverse induction on filtration degree $s$ in $H^{n}(A)$. Let $y \in F^{n} H^{n}(A)$. Then $y \in H^{n}(B)$, which is bigenerated. Now let $y \in F^{s} H^{n}(A)$, where $s<n$, and assume $F^{i} H^{n}(A)$ is bigenerated for $i>s$. Projecting $y$ to $F^{s / s+1} H^{n}(A) \simeq E_{\infty}^{s, n-s}, \rho_{s}(y)$ can be written as a sum of products $f_{i} \lambda_{i_{1}} \lambda_{i_{2}} \cdots \lambda_{i_{n}}$, where $f_{i} \in k$ and $\lambda_{i_{j}} \in S^{\prime}$. So $y$ is a sum of products $f_{i} \tilde{\lambda}_{i_{1}} \cdots \tilde{\lambda}_{i_{n}}$ and a term $\gamma$, where $\tilde{\lambda}_{i_{j}} \in T^{\prime}$ are lifts of $\lambda_{i_{j}}$ under $\rho$, and $\gamma \in F^{s+1} H^{n}(A)$. By induction, $\gamma$ is also a linear combination of products of elements from $T^{\prime}$. Thus $y$ is of the desired form.

\section{Hopf Algebras ARISING From $p$-GROUPS}

The mod- $p$ lower central series of a group $G$ gives rise to a restricted Lie algebra $L$, whose universal restricted enveloping algebra $V L$ is a Hopf algebra. Starting with a central extension of $p$-groups $H \rightarrow G \rightarrow Q$, we take enveloping algebras of the associated Lie algebras and get a central extension of Hopf algebras $F \rightarrow A \rightarrow B$. In Section 4.2 we show that $A$ is semi-Koszul if $Q$ is abelian and $H \simeq \mathbb{Z} /(p)$. In Section 4.3, we generalize to extensions of Hopf algebras $F \rightarrow A \rightarrow B$ which do not necessarily arise from extensions of $p$-groups. We show that $E^{0} A$ is semi-Koszul if $B$ is commutative.

4.1. Definitions. Let $G$ be a group, and let $G=F^{1} G \supseteq F^{2} G \supseteq \cdots$ be a descending sequence of subgroups (also called a filtration) with $F^{i} G \unrhd F^{i+1} G, F^{i} G / F^{i+1} G$ abelian. Let gr. ${ }^{F} G=\bigoplus_{i} F^{i} G / F^{i+1} G$ be the associated graded object, and let $\operatorname{gr}_{i}{ }^{F} G=F^{i} G / F^{i+1} G$. When the filtration is understood, we shall simply write gr. $G$ and $\operatorname{gr}_{i} G$. Recall the natural surjection $\rho_{i}: F^{i} G \rightarrow \operatorname{gr}_{i} G$.

For $H, K$ subgroups of $G$, let $(H, K)$ be the subgroup generated by all commutators of the form $(h, k)=h^{-1} k^{-1} h k$ for $h \in H, k \in K$. Let $H^{n}$ be the subgroup generated by $h^{n}$ for $h \in H$. An $N$-sequence is a normal series $G=G_{1} \unrhd G_{2} \unrhd G_{3} \unrhd \cdots$ such that $\left(G_{i}, G_{j}\right) \subset G_{i+j}\left[9\right.$. The successive quotients $\operatorname{gr}_{i} G=G_{i} / G_{i+1}$ of an $N$ sequence are abelian, since $\left(G_{i}, G_{i}\right) \subset G_{2 i} \subset G_{i+1}$. The associated graded abelian group gr. $G=\bigoplus_{n} G_{n} / G_{n+1}$ has the structure of a Lie algebra over $\mathbb{Z}$, with bracket $\left[\rho_{r} x, \rho_{s} y\right]=\rho_{r+s}((x, y))$. A $p$-restricted $N$-sequence is an $N$-sequence $\left\{G_{n}\right\}$ such that $G_{n}{ }^{p} \subset G_{p n}$. The associated graded group gr. $G$ is then a vector space over $\mathbb{F}_{p}$. We can define a restriction $\xi\left(\rho_{r}(x)\right)=\rho_{p r}\left(x^{p}\right)$ on gr. $G$ which makes it into a restricted Lie algebra over $\mathbb{F}_{p}$.

The fastest descending $p$-restricted $N$-sequence of $G$ is the mod-p lower central series $\left\{\Gamma^{i} G\right\}$ (see [9], [5]):

$$
\Gamma^{2 n-1} G=\Gamma^{2 n} G=\left\langle\left(\gamma_{1}, \ldots, \gamma_{r}\right)^{p^{u}} \text { such that } r \cdot p^{u} \geq n\right\rangle,
$$

where $\left(\gamma_{1}, \ldots, \gamma_{r}\right)=\left(\gamma_{1},\left(\ldots\left(\gamma_{r-2},\left(\gamma_{r-1}, \gamma_{r}\right)\right) \ldots\right)\right)$. The associated object gr. ${ }^{\Gamma} G$ is thus a restricted Lie algebra over $\mathbb{F}_{p}$. Let $\mathcal{L}(G)=\mathcal{L} G=\operatorname{gr} .{ }^{\Gamma} G$. It can easily be checked that $\mathcal{L}$ is a functor from the category of groups to the category of restricted Lie algebras over $\mathbb{F}_{p}$.

The definition we have given here is slightly different from that of [15] or [5]. Our $\Gamma^{2 n}$ equals their $\Gamma^{n}$. We have doubled the filtration degree in order not to have to worry about signs for $p$ odd.

Let $k G$ be the group ring of $G$ over $k$, with augmentation $\epsilon(g)=1$ for $g \in$ $G$. A diagonal $\psi: k G \rightarrow k G \otimes k G$ is induced by the map $g \rightarrow g \otimes g$, giving 
$k G$ the structure of a cocommutative Hopf algebra over $k$. Let $I=\operatorname{ker} \epsilon$ be the augmentation ideal of $k G$. Filter $k G$ as follows: $F^{2 n-1}=F^{2 n}=I^{n}$ for $n>0$. The associated graded algebra $E^{0}(k G)=\operatorname{gr}^{F} k G$ has the structure of a restricted Lie algebra over $k$ inherited from the multiplication in $k G$. Specifically, $\left[\rho_{r} x, \rho_{s} y\right]=$ $\rho_{r+s}(x y-y x), \xi\left(\rho_{r} x\right)=\rho_{r p}\left(x^{p}\right)$. Since $\psi$ respects the filtration on $k G$, it induces a cocommutative diagonal on $E^{0}(k G)$. It is clear that $E^{0}(k G)$ is generated as an algebra by $E_{2}^{0}(k G)=I / I^{2}$. These elements are all primitive by reason of dimension. Thus $E^{0}(k G)$ is primitively generated, and by a theorem of Milnor-Moore [12, Theorem 6.11], $E^{0}(k G)$ is isomorphic to the restricted universal enveloping algebra of its restricted Lie subalgebra $L=E_{2}^{0} k G$.

The map $\psi: \mathcal{L} G \rightarrow E^{0}(k G)$ given by $\rho_{r}(x) \mapsto \rho_{r}(x-1)$ is a restricted Lie algebra homomorphism. In fact, the image of $\psi$ lies in the primitive elements $\mathcal{P}\left(E^{0}(k G)\right)$ of $E^{0}(k G)$.

Theorem 4.2 ([15, Theorem 1.4]). The induced map

$$
\mathcal{L} G \longrightarrow \mathcal{P}\left(E^{0}(k G)\right)
$$

is an isomorphism of restricted Lie algebras.

Therefore,

$$
\mathcal{V}(\mathcal{L} G) \simeq \mathcal{V}\left(\mathcal{P}\left(E^{0}(k G)\right)\right) \simeq E^{0}(k G)
$$

as Hopf algebras over $k$.

Let $\mathcal{V} \mathcal{L} G$ denote $\mathcal{V}\left(\right.$ gr. $\left.{ }^{\Gamma} G\right)$. Studying the cohomology $H^{*}\left(E^{0}(k G)\right)$ over $k$ is thus equivalent to studying $H^{*}(\mathcal{V} \mathcal{L} G)$. The advantage of working with cohomology of Lie algebras is that the Steenrod operations $\widetilde{S q}^{0} \equiv 0, \widetilde{\mathcal{P}}^{0} \equiv 0$ there $([14]$, [11. Theorem 8.5]), which greatly simplifies the situation.

We shall need the following result.

Lemma 4.4. Let $G$ be a finite abelian p-group. Then $\mathcal{V L} G$ is a semi-Koszul algebra.

Proof. Let $G=\mathbb{Z} /\left(p^{n}\right)$. It is clear that

$$
\mathcal{V} \mathcal{L} G \simeq E^{0}\left(\mathbb{F}_{p} G\right) \simeq \mathbb{F}_{p}[x] /\left(x^{p^{n}}\right) .
$$

For a general finite $p$-group $G=\mathbb{Z} /\left(p^{n_{1}}\right) \times \cdots \times \mathbb{Z} /\left(p^{n_{k}}\right)$, then

$$
\mathcal{V} \mathcal{L} G \simeq \mathbb{F}_{p}\left[x_{1}, \ldots, x_{k}\right] /\left(x_{i}^{p^{n_{i}}}\right) .
$$

Thus

$$
H^{*}(\mathcal{V} \mathcal{L} G) \simeq \Lambda\left[z_{1}, \ldots, z_{k}\right] \otimes \mathbb{F}_{p}\left[e_{1}, \ldots, e_{k}\right]
$$

where $z_{i} \in H^{1,2}, e_{i} \in H^{2,2 p^{n_{i}}}$. If $p=2$ and some $n_{i}=1$, replace $\Lambda\left[z_{i}\right] \otimes \mathbb{F}_{p}\left[e_{i}\right]$ by $\mathbb{F}_{2}\left[z_{i}\right]$.

4.2. Extensions of $p$-groups. Let $G$ be a non-trivial finite $p$-group, and let $k=$ $\mathbb{F}_{p}$. There is an element $x$ of order $p$ in the center of $G$. Let $H \simeq \mathbb{Z} /(p)$ be the subgroup generated by $x$. We have the central extension

$$
H \stackrel{f}{\longrightarrow} G \stackrel{g}{\longrightarrow} Q=G / H
$$

Our plan is to apply the $\mathcal{L}$ functor, and then the $\mathcal{V}$ functor, to (4.5) in the hope of obtaining an exact sequence of Hopf algebras of the form (3.1). 
First, note that $g\left(\Gamma^{n} G\right)=\Gamma^{n} Q$. Refilter $H$ by $F^{n} H=f^{-1}\left(\Gamma^{n} G\right)$. According to Theorem 2.4 of [9], the following is an exact sequence of graded Lie algebras over $\mathbb{Z}$ :

$$
\text { gr. }{ }^{F} H \stackrel{\bar{f}}{\longrightarrow} \text { gr. } G \stackrel{\bar{g}}{\longrightarrow} \text { gr. } Q .
$$

Now gr. ${ }^{F} H$ is generated by $\bar{x} \in F^{2 n / 2 n+1} H$, where $2 n$ is the filtration degree of $f(x)$ in $G$. Since $\mathcal{L} H$ is generated by $\bar{x} \in \Gamma^{2 / 3} H$, gr. ${ }^{F} H \simeq \mathcal{L} H$ as ungraded Lie algebras. The sequence (4.6) is actually a sequence of graded restricted Lie algebras and - if we regrade $\mathcal{L} H$ appropriately - is isomorphic to

$$
\mathcal{L} H \longrightarrow \mathcal{L} G \longrightarrow \mathcal{L} Q .
$$

Now let

$$
L_{1} \stackrel{i}{\longrightarrow} L_{2} \stackrel{\pi}{\longrightarrow} L_{3}
$$

be an exact sequence of graded restricted Lie algebras over $\mathbb{F}_{p}$. Recall that $V L_{i}$ is defined to be the quotient of $\operatorname{Tens}\left(L_{i}\right)$ by some specific ideal $J_{i}$. It is clear that $\pi\left(J_{2}\right)=J_{3}$, and that $i^{-1}\left(J_{2}\right)=J_{1}$. Therefore,

$$
\operatorname{Tens}\left(L_{1}\right) / J_{1} \longrightarrow \operatorname{Tens}\left(L_{2}\right) / J_{2} \longrightarrow \operatorname{Tens}\left(L_{3}\right) / J_{3}
$$

is an exact sequence of Hopf algebras over $k$.

We have just proven

Proposition 4.10. Let $H, G$, and $Q$ be as in 4.5. Regrade $\mathcal{V} \mathcal{L} H$ as in (4.7). Then the following sequence of Hopf algebras is exact:

$$
\mathcal{V L} H \longrightarrow V \mathcal{L} G \longrightarrow V \mathcal{L} Q \text {. }
$$

Since $\mathcal{V} \mathcal{L} H \simeq \mathbb{F}_{p}[x] /\left(x^{p}\right)$, the central extension (4.11) is of the form $F \rightarrow A \rightarrow B$, as in (3.1). Associated to this extension is the Cartan-Eilenberg spectral sequence (3.2):

$$
\begin{aligned}
& E_{2} \simeq H^{*}(\mathcal{V} \mathcal{L} Q) \otimes \Lambda[z] \otimes \mathbb{F}_{p}[e] \Rightarrow H^{*}(\mathcal{V} \mathcal{L} G), \quad p \text { odd }, \\
& E_{2} \simeq H^{*}(\mathcal{V} \mathcal{L} Q) \otimes \mathbb{F}_{2}[z] \Rightarrow H^{*}(\mathcal{V} \mathcal{L} G), \quad p=2 \text {. }
\end{aligned}
$$

Let us now examine conditions on the group $G$ which will assure that the assumptions of Theorem 3.3 are satisfied.

Theorem 4.13. In the spectral sequence (4.12), assume $\mu=d_{2}(z)$ is a sum of products of one-dimensional elements. That is, assume $\mu$ is decomposable. Assume also that conditions (2) and (3) of Theorem 3.3 hold. Then $\mathcal{V} \mathcal{L} G$ is semi-Koszul.

Proof. This result follows from the fact that

$$
\begin{aligned}
& \widetilde{\beta \mathcal{P}}^{0}(\sigma \tau)=\widetilde{\beta \mathcal{P}}^{0}(\sigma) \widetilde{\mathcal{P}}^{0}(\tau)+\widetilde{\mathcal{P}}^{0}(\sigma) \widetilde{\beta \mathcal{P}}^{0}(\tau)=0 \text { and } \\
& \widetilde{S q}^{1}(\sigma \tau)=\widetilde{S q}^{1}(\sigma) \widetilde{S q}^{0}(\tau)+\widetilde{S q}^{0}(\sigma) \widetilde{S q}^{1}(\tau)=0,
\end{aligned}
$$

since $\widetilde{\mathcal{P}}^{0} \equiv 0$ and $\widetilde{S q}^{0} \equiv 0$ on the cohomology of a Lie algebra. Condition (1) of Theorem 3.3 is thus satisfied.

Theorem 4.15. If $Q$ is abelian, then $\mathcal{V L} G$ is semi-Koszul. 
Proof. By Lemma $4.4 H^{*}(\mathcal{V} \mathcal{L} Q)$ is isomorphic to a tensor product of algebras of the form $\Lambda\left[z_{i}\right] \otimes \mathbb{F}_{p}\left[e_{i}\right]$ (and $\mathbb{F}_{2}[z]$ for $p=2$ ), so $H^{2}\left(\mathcal{V} \mathcal{L} Q\right.$ ) is spanned by $\left\{z_{i} z_{j}, e_{i}\right\}$. Now $\widetilde{\beta \mathcal{P}}^{0}\left(z_{i} z_{j}\right)=0=\widetilde{S q}^{1}\left(z_{i} z_{j}\right)$ by (4.14) and $\widetilde{\beta \mathcal{P}}^{0}\left(e_{i}\right)=0$ by reason of internal degree. Thus condition (1) of Theorem 3.3 is satisfied. Condition (2) is satisfied because the annihilator ideal of $H^{2}(\mathcal{V L} Q)$ is generated by one-dimensional elements $\left\{z_{i}\right\}$. Finally, condition $(3)$ is satisfied since $H^{*}(\mathcal{V} \mathcal{L} Q)$ is bigenerated.

4.3. Generalization to Hopf algebras. The results in $\$ 4.2$ can be generalized somewhat to Hopf algebras. Let $A$ be a Hopf algebra over $\mathbb{F}_{p}$. Assume $A$ contains a central, primitive element $x$ of height $p$. This means $x$ anticommutes with every element of $A, x^{p}=0$, and $x^{i} \neq 0$ if $i<p$. If $p$ is odd, $x$ is forced to have even internal degree. Let $M=\mathbb{F}_{p}[x] /\left(x^{p}\right)$ be the central sub-Hopf algebra of $A$ generated by $x$.

We have the central extension of graded Hopf algebras

$$
M \stackrel{f}{\longrightarrow} A \stackrel{g}{\longrightarrow} B .
$$

For an augmented algebra $C$, let $\left\{F^{2 n-1} C=F^{2 n} C=(I C)^{n}\right\}$ be the modified augmentation ideal filtration, and let $E^{0} C=$ gr. ${ }^{F} C$. Since $\epsilon^{B}(g(y))=\epsilon^{A}(y)$, we see that $g(I A) \subset I B$, and more generally, $g\left(I A^{n}\right) \subset I B^{n}$. Thus $g$ respects the augmentation ideal filtration, and we can form the maps

$$
\begin{gathered}
\bar{g}_{2 n}: F^{2 n / 2 n+1} A=I A^{n} / I A^{n+1} \longrightarrow I B^{n} / I B^{n+1}=F^{2 n / 2 n+1} B, \\
E^{0} g: E^{0} A \longrightarrow E^{0} B .
\end{gathered}
$$

Each $\bar{g}_{2 n}$ is a map of graded modules, while $E^{0} A$ and $E^{0} B$ are bigraded algebras (by internal degree and filtration degree). It is easy to check that $E^{0} g$ is surjective. If $\bar{g}_{2 n}(\bar{y})=0$, this means $g(y) \in F^{2 n+1} B$. Since $g$ is surjective, $y$ can be written as $y=a+b$, where $a \in F^{2 n+1} A$ and $b \in \operatorname{ker} g \cap F^{2 n} A$. Since $\operatorname{ker} g=\operatorname{im} f, b=f\left(b^{\prime}\right)$ for some $b^{\prime} \in M$. Unfortunately, we cannot tell if $b^{\prime} \in F^{2 n} M$. If we refilter $M$ by $\hat{F}^{2 n} M=f^{-1}\left(F^{2 n} A\right)$, we obtain the exact sequence of bigraded algebras

$$
\text { gr. }{ }^{\hat{F}} M \longrightarrow E^{0} A \longrightarrow E^{0} B .
$$

In our case, gr. ${ }^{\hat{F}} M \simeq E^{0} M \simeq M$, except that the generator of gr. ${ }^{\hat{F}} M$ has a filtration degree equal to the filtration degree of $f(x)$ in $A$. With the understanding that $M$ is regraded as such, let us investigate the extension

$$
M \longrightarrow E^{0} A \longrightarrow E^{0} B
$$

of primitively generated Hopf algebras with associated Cartan-Eilenberg spectral sequence

$$
E_{2}^{s, t} \simeq H^{s}\left(E^{0} B\right) \otimes H^{t}(M) \Longrightarrow H^{s+t}\left(E^{0} A\right) .
$$

We recall that a Hopf algebra $C$ is monogenic if it is generated by one element $x$.

Proposition 4.18 ([12]). A monogenic Hopf algebra over $\mathbb{F}_{p}$ has the form

(1) An exterior algebra $\Lambda[x]=\mathbb{F}_{p}[x] /\left(x^{2}\right)$, with $\operatorname{deg} x$ odd for $p$ odd.

(2) A truncated polynomial algebra $A=\mathbb{F}_{p}[x] /\left(x^{p^{n}}\right)$ as in (A.6).

(3) A polynomial algebra $\mathbb{F}_{p}[x]$ with $\operatorname{deg} x$ even for $p$ odd.

In all cases, $x$ is primitive by reason of dimension. We have excluded the case $p=2, n=1$ from $(2)$, as $\mathbb{F}_{2}[x] /\left(x^{2}\right) \simeq \Lambda[x]$. 
Lemma 4.19 ([17, Proposition 2.2]). For each monogenic Hopf algebra $C$ above,

(1) $H^{*}(C) \simeq \mathbb{F}_{p}[z]$, with $z \in H^{1, \operatorname{deg} x}$.

(2) $H^{*}(C) \simeq \Lambda[z] \otimes \mathbb{F}_{p}[e]$, with $z \in H^{1, \operatorname{deg} x}, e \in H^{2, p^{n} \operatorname{deg} x}$.

(3) $H^{*}(C) \simeq \Lambda[z]$, with $z \in H^{1, \operatorname{deg} x}$.

Theorem 4.20 (Borel, [12]). A locally finite, connected, primitively generated, commutative Hopf algebra over $\mathbb{F}_{p}$ is isomorphic to a tensor product of monogenic Hopf algebras.

Theorem 4.21. Let $M, A, B$ be as in (4.16). If $B$ is commutative, then $E^{0} A$ is semi-Koszul.

Proof. The fiber $M$ has cohomology generated by $z \in H^{1}(M)$ and possibly $e \in$ $H^{2}(M)$. The element $e$ is related to $z$ by the Steenrod operation $\theta$, where $\theta$ is one of $\widetilde{\beta \mathcal{P}}^{0}$ or $\widetilde{S q}^{1}$. The spectral sequence (4.17) will collapse if $d_{3}(e)=\theta\left(d_{2}(z)\right)=0$. Since $B$ is commutative, so is $E^{0} B$. By Borel's theorem, $E^{0} B \simeq \otimes_{i} B_{i}$, where the $B_{i}$ are monogenic Hopf algebras. Then $H^{*}\left(E^{0} B\right) \simeq H^{*}\left(B_{1}\right) \otimes \cdots \otimes H^{*}\left(B_{n}\right) \simeq$ $\Lambda\left[z_{i}\right] \otimes \mathbb{F}_{p}\left[z_{j}^{\prime}\right] \otimes \mathbb{F}_{p}\left[e_{k}\right]$ with $z_{i}, z_{j}^{\prime} \in H^{1}, e_{k} \in H^{2}$. We can show $\theta \equiv 0$ on $H^{2}\left(E^{0} B\right)$ by using the same argument as in Theorem 4.15 .

Remark. A theorem of Wilkerson [17] states that a finite-dimensional, graded, connected, cocommutative Hopf algebra $A \neq k$ always contains a non-trivial central monogenic sub-Hopf algebra $C$. In the case that $C$ is a truncated polynomial algebra of height $p^{n}$, we can always find a central primitive element $x \in A$ of height $p$. The conditions of Theorem 4.21 then hold.

\section{RANK TWO $p$-GROUPS}

In this section we show that the cohomology of the Lie algebra associated with a rank $2 p$-group for $p \geq 5$ is bigenerated. A rank $2 p$-group is a finite $p$-group whose largest elementary abelian subgroup has dimension 2 . In the case $p \geq 5$, Blackburn [3] has classified these as belonging to one of the following four families: abelian, metacyclic, $C(r)$ and $G(r, e)$ of order $p^{r}$. A metacyclic $p$-group $P$ is an extension of a cyclic group by a cyclic group. A presentation for $C(r)$ is given by

$$
C(r)=\left\langle a, b, c \mid a^{p}=b^{p}=c^{p^{r-2}}=1,(a, b)=c^{p^{r-3}}, c \in \mathcal{Z}(C)\right\rangle,
$$

where $r \geq 3,(a, b)=a^{-1} b^{-1} a b$, and $\mathcal{Z}(C)$ is the center of $C$. A presentation for $G(r, e)$ is given by

$$
G(r, e)=\left\langle f, g, h \mid f^{p}=g^{p^{r-2}}=h^{p}=(g, h)=1,\left(f, h^{-1}\right)=g^{e p^{r-3}},(f, g)=h\right\rangle,
$$

where $r \geq 4, e$ is 1 or a quadratic non-residue $\bmod p$.

These also define rank $2 p$-groups for $p=3$. We include the families for $p=3$ in the calculations to follow because they are the source of most of the interesting behavior.

We will be performing explicit calculations in the cobar complex and with Massey products. See the Appendix for a review of these concepts.

5.1. $G$ of type $C(r)$. Since $c^{p^{r-3}}$ is a central element of order $p$, we have the central extension

$$
\mathbb{Z} /(p) \longrightarrow G \longrightarrow \mathbb{Z} /(p) \times \mathbb{Z} /(p) \times \mathbb{Z} /\left(p^{r-3}\right) .
$$

As the base is abelian, Theorem 4.15 tells us that $\mathcal{V} \mathcal{L} G$ is semi-Koszul. We shall now explicitly calculate $H^{*}(\mathcal{V} \mathcal{L} G)$. 
5.1.1. Case 1: $r \geq 4$.

$$
\begin{array}{rlrl}
\Gamma^{2} & =\langle a, b, c\rangle=G & \Gamma^{2 / 3} & =\mathbb{F}_{p}\langle\bar{a}, \bar{b}, \bar{c}\rangle \\
\Gamma^{2 p} & =\left\langle c^{p}\right\rangle \simeq \mathbb{Z} /\left(p^{r-3}\right) & \Gamma^{2 p / 2 p+1} & =\mathbb{F}_{p}\left\langle\overline{c^{p}}\right\rangle \\
\vdots & \vdots \\
\Gamma^{2 p^{r-3}} & =\left\langle c^{p^{r-3}}\right\rangle \simeq \mathbb{Z} /(p) & & \\
& \Gamma^{2 p^{r-3} / 2 p^{r-3}+1} & =\mathbb{F}_{p}\left\langle\overline{c^{p^{r-3}}}\right\rangle \\
\Gamma^{i / i+1} & =0 \text { for } i \neq 2 p^{j}, j \leq r-3 .
\end{array}
$$

Since $a^{-1} b^{-1} a b=c^{p^{r-3}} \in \Gamma^{2 p^{r-3}}$ falls below $\Gamma^{4}, \mathcal{L} G$ is an abelian restricted Lie algebra over $\mathbb{F}_{p}$. Moreover, $\xi(\bar{a})=\xi(\bar{b})=0, \xi\left(\overline{c^{p^{i}}}\right)=\overline{c^{p^{i+1}}}$. Let $x=\bar{a}, y=\bar{b}, z_{i}=$ $\overline{c^{p^{i-1}}}$ for $1 \leq i \leq r-2$. Then

$$
\begin{aligned}
\mathcal{V} \mathcal{L} G & =\operatorname{Tens}\left\{x, y, z_{i}\right\} /\left(\begin{array}{c}
x^{p}=y^{p}=0, z_{i}^{p}=z_{i+1} \\
z_{r-2}^{p}=0, x, y, z_{i} \text { commute }
\end{array}\right) \\
& \simeq \mathbb{F}_{p}[x, y, z] /\left(x^{p}, y^{p}, z^{p^{r-2}}\right) \quad \text { as Hopf algebras. }
\end{aligned}
$$

So $H^{*}(\mathcal{V} \mathcal{L} C(r)) \simeq \Lambda[x, y, z] \otimes \mathbb{F}_{p}[u, v, w]$, where $x, y, z \in \operatorname{Ext}^{1,2}$, and $u, v \in \operatorname{Ext}^{2,2 p}$ and $w \in E x t^{2,2 p^{r-2}}$. As usual, the one-dimensional classes $x, y$ are related to the two-dimensional classes $u, v$ by the algebraic Bockstein $-\widetilde{\beta \mathcal{P}}^{0}$, and $w=\langle z\rangle^{p^{r-2}}$. See Appendix A.3 for details.

5.1.2. Case 2: $r=3$. Let $G=C(3)=\langle a, b, c| a^{p}=b^{p}=c^{p}=1,(a, b)=c \in$ $Z(G)\rangle$. This case is more interesting, as the filtration is different. The filtration degree of $c$ is 4 , which makes $\mathcal{L} G$ non-abelian: $[\bar{a}, \bar{b}]=\bar{c} \in \Gamma^{4 / 5}$. We still have $\xi(\bar{a})=\xi(\bar{b})=0$. Thus $\mathcal{V} \mathcal{L} G \simeq \operatorname{Tens}\{a, b, c\} / J$, where $J$ is the ideal generated by the relations $\left\{a b-b a=c, a c=c a, b c=c b, a^{p}=b^{p}=c^{p}=0\right\}$. The central extension of groups

$$
\mathbb{Z} /(p) \underset{z \mapsto c}{\longrightarrow} G \underset{a, b \mapsto x, y}{\longrightarrow} \mathbb{Z} /(p) \times \mathbb{Z} /(p)
$$

induces the central extension of algebras

$$
\mathbb{F}_{p}[z] /\left(z^{p}\right) \underset{z \mapsto c}{\longrightarrow} V L G \underset{a, b \mapsto x, y}{\longrightarrow} \mathbb{F}_{p}[x, y] /\left(x^{p}, y^{p}\right)
$$

which we abbreviate as $F \longrightarrow A \longrightarrow B$. This sequence will be exact as graded algebras if we set $\operatorname{deg} z=4$. The induced Cartan-Eilenberg spectral sequence has $E_{2}$ term

$$
E_{2}^{*, *}(C(3))=H^{*}(B) \otimes H^{*}(F) \simeq \Lambda[\sigma, \tau] \otimes \mathbb{F}_{p}[\mu, \nu] \otimes \Lambda[\zeta] \otimes \mathbb{F}_{p}[\epsilon],
$$

$$
\begin{aligned}
& \sigma, \tau \in E_{2}^{1,0,2}, \quad \mu, \nu \in E_{2}^{2,0,2 p}, \quad \mu=-\widetilde{\beta \mathcal{P}}^{0}(\sigma)=\langle\sigma\rangle^{p}, \quad \nu=-\widetilde{\beta \mathcal{P}}^{0}(\tau)=\langle\tau\rangle^{p}, \\
& \zeta \in E_{2}^{0,1,4}, \quad \epsilon \in E_{2}^{0,2,4 p}, \quad \epsilon=-\widetilde{\beta \mathcal{P}}^{0}(\zeta)=\langle\zeta\rangle^{p} \text {. }
\end{aligned}
$$

The third superscript is the internal degree. The elements $\sigma \otimes 1$ and $\tau \otimes 1$ in $E_{2}^{1,0}$ are permanent cycles converging to non-zero classes $\alpha$ and $\beta$ in $H^{1,2}(A)$. Put a reverse lexicographic order on $A: c>b>a$. Choose the basis for $A$ induced by this ordering: $\mathcal{B}=\left\{c^{i} b^{j} a^{k} \mid 0 \leq i, j, k \leq p-1\right\}$. The non-trivial relations are generated by the following:

$$
a b=b a+c, \quad a c=c a, \quad b c=c b, \quad a^{p}=b^{p}=c^{p}=0 .
$$


Let $\mathcal{B}^{*}=\left\{x^{*} \mid x \in \mathcal{B}\right\}$ denote the dual basis, i.e.,

$$
\left\langle x^{*}, y\right\rangle= \begin{cases}1 & \text { if } y=x, \\ 0 & \text { otherwise. }\end{cases}
$$

Identify $\alpha, \beta$ with the classes $\left[a^{*}\right],\left[b^{*}\right]$ in the cobar complex $C^{*}(A)$. Now, $\delta\left[c^{*}\right]=$ $\left[a^{*} \mid b^{*}\right] \Rightarrow \alpha \beta=0$ in $E_{\infty}^{2,0}$. This implies

$$
d_{2}(1 \otimes \zeta)=\lambda \cdot \sigma \otimes \tau
$$

for some $\lambda \in \mathbb{F}_{p}^{\times}$, because that is the last differential which can hit $E_{2}^{2,0}$. We can ignore the constant $\lambda$; all that matters is that it is non-zero. Since $\sigma \otimes \tau=$ $1 \otimes \sigma \cdot 1 \otimes \tau$ is a product, (4.14) implies that the spectral sequence (5.3) collapses at $E_{3}$. Moreover, it can be easily seen that

(1) $d_{2}: \operatorname{Tens}(\mu, \nu) \otimes \zeta \epsilon^{k} \longrightarrow \operatorname{Ideal}(\sigma \tau) \otimes \epsilon^{k}$ is never zero,

(2) $d_{2} \equiv 0$ on $\operatorname{Ideal}(\sigma, \tau) \otimes \zeta \epsilon^{k} \subset E_{2}^{*, 2 k+1}$,

(3) $d_{2} \equiv 0$ on the $\epsilon^{k}$ lines.

The permanent classes

$$
S=\{\sigma \otimes 1, \tau \otimes 1, \mu \otimes 1, \nu \otimes 1, \sigma \otimes \zeta, \tau \otimes \zeta, 1 \otimes \epsilon\}
$$

in $E_{\infty}$ converge to elements

$$
T=\left\{\alpha, \beta u, v, x_{1}, x_{2}, e\right\}
$$

in $H^{*}(A)$, with $\alpha, \beta \in H^{1,2}(A), u, v \in H^{2,2 p}(A), x_{1}, x_{2} \in H^{2,6}(A)$ and $e \in$ $H^{2,4 p}(A)$. Every element in $E_{\infty}$ can be expressed as a sum of products from $S$, for example, $\sigma \mu^{i} \nu^{j} \otimes \zeta \epsilon^{k}=(\sigma \otimes \zeta)(\mu \otimes 1)^{i}(\nu \otimes 1)^{j}(1 \otimes \epsilon)^{k}$. Therefore,

Theorem 5.7. $T$ generates $H^{*}(A)=H^{*}(\mathcal{V L} C(3))$ multiplicatively.

We shall look at the relations in $H^{*}(A)$ in the following section.

5.2. Relations in $H^{*}(\mathcal{V L C}(3))$. We will use a theorem of Ravenel 16, Appendix 1.4] on Massey products in spectral sequences. Refer to A.2 for the definition of Massey products. The formulation is somewhat complicated, but the main idea, due to Kahn [6], is that we do not want any higher "cross-differentials" to interfere with $d_{2}$. Since $d_{r}=0$ for $r \geq 3$, this is not an issue. Thus we can safely state that if $a_{i} \in E_{2}^{*, *}$ converge to $u_{i}$ in $H^{*}(A)$, then the Massey product $\left\langle a_{1}, \ldots, a_{n}\right\rangle \in E_{3}$ (when defined) will converge to $\left\langle u_{1}, \ldots, u_{n}\right\rangle$, up to indeterminacy, of course. We shall also make use of the following "juggling theorem":

Lemma 5.8 ([16, A1.4.6]). If $\left\langle u_{1}, u_{2}, u_{3}\right\rangle$ and $\left\langle u_{2}, u_{3}, u_{4}\right\rangle$ are defined, then

$$
u_{1}\left\langle u_{2}, u_{3}, u_{4}\right\rangle=\left\langle\bar{u}_{1}, \bar{u}_{2}, \bar{u}_{3}\right\rangle u_{4}
$$

where $\bar{u}=(-1)^{\operatorname{deg} u+1} u$ and $\operatorname{deg} u$ means total degree of $u$.

Proposition 5.9. In $H^{*}(\mathcal{V} \mathcal{L} C(3))$, the multiplicative relations are generated by the following:

(1) $\alpha^{2}=\alpha \beta=\beta^{2}=0$.

(2) $\alpha x_{2}=-\beta x_{1}$.

(3) $\alpha x_{1}=\left\{\begin{array}{ll}\beta u, & p=3, \\ 0, & p>3,\end{array} \quad \beta x_{2}= \begin{cases}-\alpha v, & p=3 \\ 0, & p>3\end{cases}\right.$ 
(4) $x_{1}^{2}, x_{1} x_{2}, x_{2}^{2}= \begin{cases}-u x_{2},-u v, v x_{1}, & p=3 \\ 0,0,0, & p>3 .\end{cases}$

In addition, we have the following Massey products:

(5) $x_{1}=\langle\alpha, \alpha, \beta\rangle=-1 / 2\langle\alpha, \beta, \alpha\rangle=\langle\beta, \alpha, \alpha\rangle$.

(6) $x_{2}=-\langle\alpha, \beta, \beta\rangle=1 / 2\langle\beta, \alpha, \beta\rangle=-\langle\beta, \beta, \alpha\rangle$.

(7) $\langle\alpha\rangle^{p}=-\widetilde{\beta \mathcal{P}}^{0}(\alpha)=u, \quad\langle\beta\rangle^{p}=-\widetilde{\beta \mathcal{P}}^{0}(\beta)=v$.

We will prove the last three first.

Proof of $5.9(5)$ and $5.9(6)$. Using the notation of $s$ A.2 let $(U, \delta)=\left(E_{2}^{* *}, d_{2}\right)$. The elements $a_{1}=a_{2}=\sigma \otimes 1, a_{3}=\tau \otimes 1$ are in $E_{2}^{1,0}$, and have total degree 1. Therefore $\overline{a_{i}}=a_{i}$. Now $a_{1} a_{2}=\sigma^{2} \otimes 1=0$, and $a_{2} a_{3}=\sigma \otimes \tau=d_{2}(1 \otimes \zeta)$, so we can take $u_{1}=0, u_{2}=1 \otimes \zeta$. Then $\left\langle a_{1}, a_{2}, a_{3}\right\rangle=\sigma \otimes 1 \cdot 1 \otimes \zeta+0 \cdot \tau \otimes 1=\sigma \otimes \tau$, which converges to $x_{1}$. The indeterminacy is $\alpha H^{1}+H^{1} \beta$, which is 0 since $H^{1}(A)=\mathbb{F}_{p}\langle\alpha, \beta\rangle$ and $\alpha^{2}=\alpha \beta=\beta^{2}=0$.

$\langle\beta, \alpha, \alpha\rangle=\tau \otimes 1 \cdot 0+(-1 \otimes \zeta) \cdot \sigma \otimes 1=\sigma \otimes \zeta$. The minus sign comes from the fact that $\beta \alpha=-\alpha \beta$. It disappears because $1 \otimes \zeta$ and $\sigma \otimes 1$ anticommute.

$\langle\alpha, \beta, \alpha\rangle=\sigma \otimes 1 \cdot(-1 \otimes \zeta)+1 \otimes \zeta \cdot \sigma \otimes 1=-2 \sigma \otimes \zeta$.

Now let $U=C^{* *}(A)$, the cobar complex of $A$. $\alpha$ is represented by $a_{1}=a_{2}=$ $\left[a^{*}\right], \beta$ by $a_{3}=\left[b^{*}\right]$. Let us calculate $\langle\alpha, \alpha, \beta\rangle$ in $U \cdot u_{1}=\left[a^{2^{*}}\right], u_{2}=\left[c^{*}\right]$, and $\left\langle a_{1}, a_{2}, a_{3}\right\rangle=\left[a^{*} \mid c^{*}\right]+\left[a^{2^{*}} \mid b^{*}\right]$. We now have a representative for $x_{1}$ in $C^{*}(A)$.

Other representatives are

$$
\begin{aligned}
& x_{1}=-\frac{1}{2}\langle\alpha, \beta, \alpha\rangle=\frac{1}{2}\left(\left[a^{*} \mid c^{*}\right]-\left[c^{*} \mid a^{*}\right]-\left[a^{*} \mid b a^{*}\right]\right), \\
& x_{1}=\langle\beta, \alpha, \alpha\rangle=\left[b^{*} \mid a^{2 *}\right]-\left[c^{*} \mid a^{*}\right]+\left[b a^{*} \mid a^{*}\right] .
\end{aligned}
$$

The procedure for $x_{2}$ is similar. Some representatives are

$$
\begin{aligned}
& x_{2}=-\langle\alpha, \beta, \beta\rangle=-\left[a^{*} \mid b^{2 *}\right]-\left[c^{*} \mid b^{*}\right], \\
& x_{2}=-\langle\beta, \beta, \alpha\rangle=-\left[b^{*} \mid b a^{*}\right]+\left[b^{*} \mid c^{*}\right]-\left[b^{2 *} \mid a^{*}\right], \\
& x_{2}=\frac{1}{2}\langle\beta, \alpha, \beta\rangle=\frac{1}{2}\left(\left[b^{*} \mid c^{*}\right]+\left[b a^{*} \mid b^{*}\right]-\left[c^{*} \mid b^{*}\right]\right) .
\end{aligned}
$$

Proof of $5.9(7)$. Follows from naturality of the algebraic Steenrod operations.

Proof of $\left[5.9(1)\right.$. Follows from relations in $E_{\infty}$.

Proof of $\overline{5.9}(2) . \alpha x_{2}=-\alpha\langle\alpha, \beta, \beta\rangle=-\langle\bar{\alpha}, \bar{\alpha}, \bar{\beta}\rangle \beta=-x_{1} \beta=-\beta x_{1}$.

Proof of $\left[5.9(3) . \alpha x_{1}=\alpha\langle\alpha, \alpha, \beta\rangle=\langle\bar{\alpha}, \bar{\alpha}, \bar{\alpha}\rangle \beta=\langle\alpha, \alpha, \alpha\rangle \beta=u \beta\right.$ for $p=3$. For larger primes, $\langle\alpha\rangle^{3}=0$, since for $\langle\alpha\rangle^{p}$ to be defined, we need all lower symmetric Massey products to vanish. Similarly, $\beta x_{2}=-\beta\langle\beta, \beta, \alpha\rangle=-\langle\beta, \beta, \beta\rangle \alpha=-v \alpha$ for $p=3$, while it vanishes for larger primes. In either situation, the products fall to the lower filtration level $F^{3} H^{3}(A)=E_{\infty}^{3,0}$.

Proof of $\left[5.9(4)\right.$. Since $(\sigma \otimes \zeta)^{2}=-\sigma^{2} \otimes \zeta^{2}=0$ in $E_{\infty}^{2,2} \simeq F^{2 / 3} H^{4}(A)=F^{2} H^{4}(A) /$ $F^{3} H^{4}(A)$, we deduce that $x_{1}^{2} \in F^{3} H^{4}(A)$. Since $F^{3} H^{4}(A)$ is spanned by $\left\{u x_{1}, u x_{2}\right.$, $\left.v x_{1}, v x_{2}, u^{2}, u v, v^{2}\right\}$, we can express $x_{1}^{2}$ as a linear combination

$$
x_{1}^{2}=c_{1} u x_{1}+c_{2} u x_{2}+c_{3} v x_{1}+c_{4} v x_{2}+c_{5} u^{2}+c_{6} u v+c_{7} v^{2}, \quad c_{i} \in \mathbb{F}_{p} .
$$


The element $x_{1}^{2}$ has internal degree 12 , while the elements on the right-hand side of (5.12) have internal degree larger than or equal to $2 p+6$. For $p>3$, we are forced to conclude that $x_{1}^{2}=0$. As usual, the case $p=3$ requires special attention.

Let $p=3$. Multiplying both sides of (5.12) by $\alpha$ gives us $\alpha x_{1}^{2}=\beta u x_{1}$ on the left, and $c_{1} \beta u^{2}-c_{2} \beta u x_{1}+c_{3} \beta u v-c_{4} \beta v x_{1}+c_{5} \alpha u^{2}+c_{6} \alpha u v+c_{7} \alpha v^{2}$ on the right. The summands are linearly independent in $H^{5}(A)$ because their representatives in $E_{\infty}$ are linearly independent. Therefore, $c_{2}=-1$, and all the other $c_{i}$ are zero. Starting with linear combinations in $H^{4}(A)$ for $x_{1} x_{2}$ and $x_{2}^{2}$, then multiplying by $\alpha$, will yield the other two identities.

These relations all come about from the fact that $\sigma^{2}=\tau^{2}=\zeta^{2}=0, \sigma \tau=-\tau \sigma$ in $E_{\infty}$. Since there are no other identities of this type in $E_{\infty}$, there are no new relations in $H^{*}(A)$.

\section{3. $G$ of type $G(r, e)$. We next turn our attention to groups $G$ of type}

$$
G(r, e)=\left\langle f, g, h \mid f^{p}=g^{p^{r-2}}=h^{p}=(g, h)=1,\left(f, h^{-1}\right)=g^{e p^{r-3}},(f, g)=h\right\rangle .
$$

We will soon see that for our purposes, we can take $e=1$.

Lemma 5.13. $g^{p} \in \mathcal{Z}(G)$.

Proof. Since $(f, g)=h$, we have $f^{-1} g^{-1} f=h g^{-1}$, and

$$
\left(f, g^{p}\right)=f^{-1} g^{-p} f g^{p}=\left(f^{-1} g^{-1} f\right)^{p} g^{p}=\left(h g^{-1}\right)^{p} g^{p}=h^{p} g^{-p} g^{p}=1 .
$$

The second-to-last equality follows because $(g, h)=1$.

We will investigate the exact sequence

$$
\mathbb{Z} /(p)\langle d\rangle \underset{d \mapsto g^{p^{r-3}}}{\longrightarrow} G(r) \longrightarrow Q(r-1) .
$$

This is a central extension by Lemma 5.13. As the base is not abelian, we cannot use Theorem 4.15 The cokernel $Q$ has generators $\tilde{f}, \tilde{g}, \tilde{h}$ and relations

$$
\tilde{f}^{p}=\tilde{h}^{p}=(\tilde{g}, \tilde{h})=1,\left(\tilde{f}, \tilde{h}^{-1}\right)=\tilde{g}^{e p^{r-3}}=1,(\tilde{f}, \tilde{g})=\tilde{h} .
$$

Therefore $Q(r-1) \simeq\left\langle a, b, c \mid a^{p}=b^{p^{r-3}}=c^{p}=1,(a, b)=c \in Z(Q)\right\rangle$, where $a=\tilde{f}$, $b=\tilde{g}, c=\tilde{h}$. For $r=4, Q(r-1)=Q(3) \simeq C(3)$. We will focus on this case presently. The case $r>4$ is taken up in Section 5.3 .2

5.3.1. Case 1: $r=4$. For the extension (5.15), we have

$$
\mathbb{Z} /(p) \underset{d \mapsto g^{p}}{\longrightarrow} G(4) \underset{f, g, h \mapsto a, b, c}{\longrightarrow} Q=C(3)
$$

with $f, g, h, d$ in filtration degree $2,2,4,2 p$, respectively. The Lie algebra $\mathcal{L} G$ is generated by $\left\{x=\bar{f}, y_{1}=\bar{g}, y_{2}=\overline{g^{p}}, z=\bar{h}\right\}$. Since $2+4=2 p \Leftrightarrow p=3,[\bar{f}, \bar{h}]=0$ for $p>3$. Since $\overline{h^{-1}}=-\bar{h},[\bar{f}, \bar{h}]=-\left[\bar{f}, \overline{h^{-1}}\right]=-\overline{g^{e p}}=-e \overline{g^{p}}$ for $p=3$. Summarizing, the non-trivial structure of $\mathcal{L} G$ is as follows:

$$
\xi\left(y_{1}\right)=y_{2}, \quad\left[x, y_{1}\right]=z, \quad[x, z]= \begin{cases}-e y_{2} & p=3, \\ 0 & p>3 .\end{cases}
$$

For $p=3, e$ can only equal 1 , so we see that $e$ does not really matter. 
Taking the usual quotient of Tens $\left\{x, y_{1}, y_{2}, z\right\}$ by the ideal $J$ of relations as in Definition 2.4

$$
\mathcal{V} \mathcal{L} G \simeq \begin{cases}\operatorname{Tens}\{f, g, h\} /\left(\begin{array}{c}
f^{p}=g^{p^{2}}=h^{p}=0, f g=h+g f \\
f g^{p}=g^{p} f, f h=h f, g h=h g
\end{array}\right) & p>3, \\
\operatorname{Tens}\{f, g, h\} /\left(\begin{array}{c}
f^{p}=g^{p^{2}}=h^{p}=0, f g=h+g f \\
f g^{p}=g^{p} f, f h=-g^{p}+h f, g h=h g
\end{array}\right) & p=3,\end{cases}
$$

under the mapping $x \mapsto f, y_{1} \mapsto g, y_{2} \mapsto g^{p}, z \mapsto h$.

The central extension of Hopf algebras,

$$
\mathbb{F}_{p}[d] /\left(d^{p}\right) \underset{d \mapsto g^{p}}{\stackrel{i}{\longrightarrow}} \mathcal{V} \mathcal{L} G \underset{f, g, h \mapsto a, b, c}{\longrightarrow} \mathcal{H} Q=\mathcal{V} \mathcal{L} C(3),
$$

denoted by $F \stackrel{i}{\longrightarrow} A \stackrel{\pi}{\longrightarrow} B$, gives us a spectral sequence

$$
E_{2}^{*, *}(G(4)) \simeq H^{*}(B) \otimes\left(\Lambda[z] \otimes \mathbb{F}_{p}[w]\right) \Rightarrow H^{*}(A),
$$

where $z \in E_{2}^{0,1,2 p}$ and $w \in E_{2}^{0,2,2 p^{2}}$. We shall suppress tensor notation in spectral sequences (e.g. $1 \otimes z=z$ ) from now on.

Proposition 5.20. In the spectral sequence (5.19)

$$
d_{2}(z)= \begin{cases}v-x_{1}, & p=3 \\ v, & p>3\end{cases}
$$

Proof. Put an ordering $h>g>f$ on $A=\mathcal{V} \mathcal{L} G$. A basis for $A$ consists of $\left\{h^{i} g^{j} f^{k} \mid 0 \leq i, k \leq p-1,0 \leq j \leq p^{2}-1\right\}$. Using the relations (5.17) for $A$, we see that in the cobar complex $C^{*}(A)$,

$$
\delta\left[g^{p *}\right]= \begin{cases}\sum_{i=1}^{p-1}\left[g^{i^{*}} \mid g^{p-i^{*}}\right]-\left[f^{*} \mid h^{*}\right]-\left[f^{2^{*}} \mid g^{*}\right] & p=3, \\ \sum_{i=1}^{p-1}\left[g^{i^{*}} \mid g^{p-i^{*}}\right] & p>3 .\end{cases}
$$

Under the edge map $\pi^{*}: H^{*}(B) \longrightarrow H^{*}(A), v \mapsto \operatorname{class}\left(\sum_{i=1}^{p-1}\left[g^{i^{*}} \mid g^{p-i^{*}}\right]\right)$ and $x_{1} \mapsto \operatorname{class}\left(\left[f^{*} \mid h^{*}\right]+\left[f^{2^{*}} \mid g^{*}\right]\right)$. The only differential that can cause the right-hand side in (5.21) to be zero is $d_{2}(z)$.

Proposition 5.22. $\widetilde{\beta \mathcal{P}}^{0}(v)=\widetilde{\beta \mathcal{P}}^{0}\left(x_{1}\right)=0$ in $H^{3}(B)$.

Proof. $v, x_{1} \in H^{2,2 p}(B)$. The image of $\widetilde{\beta \mathcal{P}}^{0}: H^{2,2 p}(B) \longrightarrow H^{3,2 p^{2}}$ is zero because the internal degrees of the elements of $H^{3}(B)$ are all less than or equal to $2+4 p$, which is less than $2 p^{2}$.

Proposition 5.23. $d_{2}(z)$ is not a zero divisor in $H^{*}(B)$.

Proof. Assume $d_{2}(z) y=0$ for some non-zero element $y \in H^{s+t}(B)$ of filtration degree $s$. Project $y$ to $\bar{y} \in F^{s / s+1} H^{s+t}(B) \simeq E_{\infty}^{s, t}(C(3))$.

For $p>3$, the equation $v y=0$ projects to $\nu \bar{y}=0$. Since $\nu$ is not a zero divisor in $E_{\infty}(C(3)), \bar{y}=0$. This means $y \in F^{s+1} H^{*}(B)$, which is a contradiction.

For $p=3$, we have $\left(v-x_{1}\right) y=0$, or $v y=x_{1} y$. This projects to $\nu \bar{y}=\sigma \zeta \bar{y}$. Note that $\nu \bar{y} \in E_{\infty}^{s+2, t}$, while $\sigma \zeta \bar{y} \in E_{\infty}^{s+1, t+1}$. The two cannot possibly be equal, unless of course they are both zero. But $\nu$ is not a zero divisor, and the same contradiction ensues. 
We conclude

Proposition 5.24. The spectral sequence (5.19) collapses at $E_{3}$, and

$$
H^{*}(A) \simeq \begin{cases}H^{*}(B) /\left(v-x_{1}\right) \otimes \mathbb{F}_{p}[w], & p=3 \\ H^{*}(B) /(v) \otimes \mathbb{F}_{p}[w], & p>3\end{cases}
$$

Theorem 5.25. $H^{*}(\mathcal{V} \mathcal{L} G(4))$ is generated by $\left\{\alpha, \beta, u, x_{1}, x_{2}, e, w\right\}$, with $\alpha, \beta \in$ $H^{1,2}, x_{1}, x_{2} \in H^{2,6}, u \in H^{2,2 p}, e \in H^{2,4 p}$, and $w \in H^{2,2 p^{2}}$. The relations are generated by

(1) $\alpha^{2}=\alpha \beta=\beta^{2}=0$.

(2) $\alpha x_{2}=-\beta x_{1}$.

(3) $\alpha x_{1}=\left\{\begin{array}{ll}\beta u, & p=3, \\ 0, & p>3,\end{array} \quad \beta x_{2}= \begin{cases}-\beta u, & p=3 \\ 0, & p>3 .\end{cases}\right.$

(4) $x_{1}^{2}, x_{1} x_{2}, x_{2}^{2}= \begin{cases}-u x_{2},-u x_{1},-u x_{2}, & p=3, \\ 0,0,0, & p>3 .\end{cases}$

In particular, $\mathcal{V} \mathcal{L} G(4)$ is semi-Koszul. The Massey products of Proposition 5.9 still hold, except now $\langle\beta\rangle^{p}=x_{1}$ for $p=3$, and $\langle\beta\rangle^{p}=0,\langle\beta\rangle^{p^{2}}=w$ for $p>3$.

Proof. This is simply a matter of setting $v=0$ or $v=x_{1}$ in the relations for $H^{*}(\mathcal{V} \mathcal{L} C(3))$ (Proposition [5.9). The class $w$ is formally the coboundary of $\left[g^{p^{2 *}}\right]$. For $p>3$, this is equal to the representative of $\langle\beta\rangle^{p^{2}}$ in the cobar complex.

Remark. Notice that $x_{2}^{2}=-u x_{2}$ implies $x_{2}+u$ is a zero divisor, with $x_{2}\left(x_{2}+u\right)=0$. If we had a central extension of 3 -groups $\mathbb{Z} /(3) \longrightarrow K \longrightarrow G(4)$ and the induced spectral sequence (4.12) had $d_{2}(z)=x_{2}+u$, then $x_{2} \otimes z \in E_{\infty}^{2,1}$ would be an indecomposable element of dimension 3 . In this case, $\mathcal{V} \mathcal{L} K$ would not be semiKoszul.

5.3.2. Case 2: $r>4$. Now let $G=G(r, e), r>4, p \geq 3$. The situation is

$$
\mathbb{Z} /(p)\langle d\rangle \underset{d \mapsto g^{p^{r-3}}}{\longrightarrow} G(r) \underset{f, g, h \mapsto a, b, c}{\longrightarrow} Q(r-1),
$$

where

$$
Q(r-1) \simeq\left\langle a, b, c \mid a^{p}=b^{p^{r-3}}=c^{p}=1,[a, b]=c \in Z(Q)\right\rangle .
$$

The filtration degrees of $f, g, h, d$ are $2,2,4,2 p^{r-3}$, respectively.

Let us first deal with finding $H^{*}(\mathcal{V} \mathcal{L} Q(r-1))$. There is a central extension for $Q$

$$
\mathbb{Z} /(p)\langle z\rangle \underset{z \mapsto c}{\longrightarrow} Q\langle a, b, c\rangle \underset{a, b \mapsto x, y}{\longrightarrow} \mathbb{Z} /(p) \times \mathbb{Z} /\left(p^{r-3}\right)\langle x, y\rangle
$$

which is very similar to (15.1). The induced algebra extension

$$
\mathbb{F}_{p}[z] /\left(z^{p}\right) \longrightarrow \mathcal{V} \mathcal{L} Q(r-1) \longrightarrow \mathbb{F}_{p}[x, y] /\left(x^{p}, y^{p^{r-3}}\right), \quad \operatorname{deg} z=4,
$$

gives us the spectral sequence

$$
E_{2}^{* *}(Q(r-1)) \simeq \Lambda[\sigma, \tau] \otimes \mathbb{F}_{p}[\mu, \nu] \otimes \Lambda[\zeta] \otimes \mathbb{F}_{p}[\epsilon]
$$

with $\sigma, \tau, \mu, \nu, \zeta, \epsilon$ as in (5.3) except $\nu$ has internal degree $2 p^{r-3}$ and $\nu=\langle\tau\rangle^{p^{r-3}}$. Just as in (5.4), $d_{2}(\zeta)=\sigma \tau$ and the spectral sequence collapses at $E_{3}$. 
Theorem 5.26. $H^{*}(\mathcal{V} \mathcal{L} Q(r-1))$ is generated by $\left\{\alpha, \beta, x_{1}, x_{2}, u, v, e\right\}$, with $\alpha, \beta \in$ $H^{1,2}, x_{1}, x_{2} \in H^{2,6}, u \in H^{2,2 p}, v \in H^{2,2 p^{r-3}}$ and $e \in H^{2,4 p}$. Relations are generated by

(1) $\alpha^{2}=\alpha \beta=\beta^{2}=0$.

(2) $\alpha x_{2}=-\beta x_{1}$.

(3) $\alpha x_{1}=\left\{\begin{array}{ll}\beta u, & p=3, \\ 0, & p>3,\end{array} \quad \beta x_{2}=0\right.$.

(4) $x_{1}^{2}, x_{1} x_{2}, x_{2}^{2}= \begin{cases}-u x_{2}, 0,0, & p=3, \\ 0,0,0, & p>3 .\end{cases}$

The Massey products of Proposition 5.9 still hold, except $\langle\beta\rangle^{p^{r-3}}=v$ for all $p$.

Proof. The proofs of (11) and (2) are the same as in 5.9 For the second half of (3), $\beta x_{2}=-\beta\langle\beta, \beta, \alpha\rangle=-\langle\beta, \beta, \beta\rangle \alpha=0$ even for $p=3$. In the proof of (4) for $p=3$, we are reduced to solving the equation

$$
x_{i} x_{j}=c_{1} u x_{1}+c_{2} u x_{2}+c_{5} u^{2}, \quad c_{k} \in \mathbb{F}_{p},
$$

by reason of internal dimension. Multiplying both sides by $\alpha$ and equating coefficients yield the desired identities. For example, $\alpha x_{2}^{2}=-\beta x_{1} x_{2}=0$, while the right side of (5.27) becomes $c_{1} \beta u^{2}-c_{2} \beta u x_{1}+c_{5} \alpha u^{2}$.

We now turn to finding $H^{*}(\mathcal{V} \mathcal{L} G(r))$. Notice that since $\left(f, h^{-1}\right)=g^{p^{r-3}}$ falls below $\Gamma^{6} G,[\bar{f}, \bar{h}]=0$ in $\mathcal{L} G$ even for $p=3$. Thus (5.17) becomes

$$
\mathcal{V L} G(r) \simeq \operatorname{Tens}\{f, g, h\} /\left(\begin{array}{c}
f^{p}=g^{p^{r-2}}=h^{p}=0, f g=h+g f \\
f g^{p}=g^{p} f, f h=h f, g h=h g
\end{array}\right) .
$$

As in (5.18), setting $\operatorname{deg} d=2 p^{r-3}$ gives us the graded algebra extension

$$
\mathbb{F}_{p}[d] /\left(d^{p}\right) \stackrel{i}{\longrightarrow} \mathcal{V} \mathcal{L} G(r) \stackrel{\pi}{\longrightarrow} \mathcal{V} \mathcal{L} Q(r-1)
$$

which induces the analogue of (5.19)

$$
E_{2}^{*, *}(G(r)) \simeq H^{*}(\mathcal{V} \mathcal{L} Q(r-1)) \otimes \Lambda[z] \otimes \mathbb{F}_{p}[w] .
$$

Proposition 5.29. In the spectral sequence (5.28), $d_{2}(z)=v$.

Proof. In the cobar complex $C^{*}(\mathcal{V} \mathcal{L} G(r)), \delta\left[g^{p^{r-3 *}}\right]=\sum_{i=1}^{p^{r-3}-1}\left[g^{i^{*}} \mid g^{p^{r-3}-i^{*}}\right]$, which is the representative of $\pi^{*}(v)$. Thus $d_{2}(z)=v$ for all $p$.

Proceeding in the same way as in Propositions 5.22, 5.23 and 5.24, we obtain

Proposition 5.30. $\widetilde{\beta \mathcal{P}}^{0}(v)=0$, and $v$ is not a zero divisor in $H^{*}(\mathcal{V} \mathcal{L} Q(r-1))$. Thus the spectral sequence (5.28) collapses at $E_{3}$ and

$$
H^{*}(\mathcal{V} \mathcal{L} G(r)) \simeq E_{\infty} \simeq H^{*}(\mathcal{V} \mathcal{L} Q(r-1)) /(v) \otimes \mathbb{F}_{p}[w] .
$$

Finally,

Theorem 5.31. $H^{*}(\mathcal{V} \mathcal{L} G(r))$ is generated by $\alpha, \beta, u, x_{1}, x_{2}, e, w$. The bidegrees of the generators are as in Theorem 5.25, except $w \in H^{2,2 p^{r-2}}$. Relations are as in Theorem 5.25 except now $\beta x_{2}=x_{1} x_{2}=x_{2}^{2}=0,\langle\beta\rangle^{p^{r-2}}=w$ for all $p$. In particular, $H^{*}(\mathcal{V} \mathcal{L} G(r))$ is semi-Koszul. 
5.4. $G$ metacyclic. Let $G$ be a metacyclic $p$-group. That is, there is an extension

$$
\mathbb{Z} /\left(p^{m}\right) \longrightarrow G \longrightarrow \mathbb{Z} /\left(p^{n}\right) .
$$

For $p$ odd, any metacyclic $p$-group $G$ has presentation

$$
G=P(m, n, q, l)=\left\langle x, y \mid x^{p^{m}}=1, y^{p^{n}}=x^{p^{q}},(x, y)=x^{p^{l}}\right\rangle
$$

for integers $m, n, l, q \geq 1$ satisfying $\left(p^{l}+1\right)^{p^{n}} \equiv 1 \bmod p^{m}$ and $\left(p^{l}+1\right) p^{q} \equiv p^{q}$ $\bmod p^{m}$ [4]. We can get all groups up to isomorphism by requiring $l \leq m, q \leq m$, $n+l \geq m$ and $q+l \geq m$. Note that $y^{p^{m+n-q}}=1$.

It is clear that $\mathcal{L} G$ is generated by $x_{i}=\overline{x^{p^{i-1}}} \in \Gamma^{2 p^{i-1} / 2 p^{i-1}+1}, 1 \leq i \leq m$, and $y_{j}=\overline{y^{p^{j-1}}} \in \Gamma^{2 p^{j-1} / 2 p^{j-1}+1}, 1 \leq j \leq n+m-q$, some of which may be zero.

Lemma 5.32. For all $i, j,\left[x_{i}, y_{j}\right]=0$.

Proof. $y^{-1} x y=x^{p^{l}+1} \Rightarrow y^{-p^{j}} x^{p^{i}} y^{p^{j}}=x^{p^{i}\left(p^{l}+1\right)^{p^{j}}} \Rightarrow\left(x^{p^{i}}, y^{p^{j}}\right)=x^{p^{p^{i}}\left(\left(p^{l}+1\right)^{p^{j}}-1\right)}$. The exponent is $p^{i}\left(\left(p^{l}+1\right)^{p^{j}}-1\right)=p^{i}\left(p^{l p^{j}}+\cdots+p^{j} p^{l}+1-1\right)=p^{i+j+l} a, \quad a \in \mathbb{Z}$. Therefore, $\left(x^{p^{i}}, y^{p^{j}}\right)=\left(x^{a}\right)^{p^{i+j+l}} \in \Gamma^{2 p^{i+j+l}} \subsetneq \Gamma^{2\left(p^{i}+p^{j}\right)}$, even for $i=j=0$.

We can break the analysis of $\mathcal{L} G$ down into three cases:

Case 1: $n<q$.

$\xi\left(y_{j}\right)=y_{j+1}$ for $j<n$. Since $y^{p^{n}}=x^{p^{q}}$ falls down to a lower filtration level, $\xi\left(y_{n}\right)=\overline{y^{p^{n}}}=0$, and there are no $y_{j}$ for $j>n$. The other generators $\left\{x_{i}\right\}_{1 \leq i \leq m}$ have restriction $\xi\left(x_{i}\right)=x_{i+1}$ for $i<m$ and $\xi\left(x_{m}\right)=0$. Then

$$
\mathcal{V} \mathcal{L} G \simeq \mathbb{F}_{p}[x, y] /\left(x^{p^{m}}, y^{p^{n}}\right) \quad \operatorname{deg} x=\operatorname{deg} y=2 .
$$

Case 2: $n>q$

By the same reasoning as above, there are no $x_{i}$ for $i>q . \xi\left(x_{i}\right)=x_{i+1}$ for $i<q$, $\xi\left(x_{q}\right)=0 . \xi\left(y_{j}\right)=y_{j+1}$ for $j<n+m-q$, and $\xi\left(y_{n+m-q}\right)=0$. So

$$
\mathcal{V} G \simeq \mathbb{F}_{p}[x, y] /\left(x^{p^{q}}, y^{p^{n+m-q}}\right) \quad \operatorname{deg} x=\operatorname{deg} y=2 .
$$

Case 3: $n=q$.

In this case, $x^{p^{q}}=y^{p^{n}}$ in $\Gamma^{2 p^{q}}=\Gamma^{2 p^{n}}$. Let $z=y x^{p^{m-n}-1}$. Then $(x, z)=$ $x^{-1} x^{1-p^{m-n}} y^{-1} x y x^{p^{m-n}-1}=x^{p^{l}}=(x, y)$.

Claim. $z^{p^{n}}=1$.

Proof. It can be shown by induction that $\left(y x^{a}\right)^{b}=y^{b} x^{a\left[\left(p^{l}+1\right)^{b}-1\right] / p^{l}}$. For $a=$ $p^{m-n}-1, b=p^{n}$, the exponent of $x$ is

$$
\left(p^{m-n}-1\right)\left(\left(p^{l}+1\right)^{p^{n}}-1\right) / p^{l}=\left(p^{m-n}-1\right) p^{n+l} c / p^{l}=\left(p^{m}-p^{n}\right) c
$$

where $c=1+\frac{1}{p^{n}}\left(\begin{array}{c}p^{n} \\ 2\end{array}\right) p^{l}+\frac{1}{p^{n}}\left(\begin{array}{c}p^{n} \\ 3\end{array}\right) p^{2 l}+\cdots=1+p^{l} d$, for some $d \in \mathbb{Z}$. Then $z^{p^{n}}=y^{p^{n}} x^{\left(p^{m}-p^{n}\right) c}=x^{p^{n}(-c+1)}=x^{p^{n+l} d}=1$ since $n+l \geq m$.

So $G \simeq\left\langle x, z \mid x^{p^{m}}=1, z^{p^{n}}=1,(x, z)=x^{p^{l}}\right\rangle$. Generators for $\mathcal{L} G$ are $\left\{x_{i}\right\}_{1 \leq i \leq m}$ and $\left\{z_{j}\right\}_{1 \leq j \leq n}$, and

$$
\mathcal{V} \mathcal{L} G \simeq \mathbb{F}_{p}[x, z] /\left(x^{p^{m}}, z^{p^{n}}\right) \quad \operatorname{deg} x=\operatorname{deg} z=2 .
$$

Note that the transformation $y \mapsto z$ in $G$ corresponds to the map $y_{1} \mapsto z_{1}=y_{1}-x_{1}$ in $\mathcal{L} G$. 
Theorem 5.33. Let $G=P(m, n, q, l)$. Then

$H^{*}(\mathcal{V} \mathcal{L} G) \simeq \Lambda[x, y] \otimes \mathbb{F}_{p}[u, v], x, y \in H^{(1,2)}, u=\langle x\rangle^{a} \in H^{(2,2 a)}, v=\langle y\rangle^{b} \in H^{(2,2 b)}$, where $a=p^{m}, b=p^{n}$ if $q \geq n$ and $a=p^{q}, b=p^{m+n-q}$ if $q<n$.

We have finally proven

Theorem 5.34. Let $p \geq 5$, and let $G$ be a finite p-group of rank 2. Then $\mathcal{V L} G$ is semi-Koszul.

\section{Appendix A.}

A.1. The cobar complex. We summarize the construction of the cobar complex of $A$, which can be used to calculate the cohomology of $A$. Refer to Priddy [13] Section 1] for more details.

As in Section 2 let $A$ be an algebra with augmentation ideal $I$. The cobar complex of $A$, denoted by $C^{*}(A)$, is given by $C^{s}(A)=\left(I^{*}\right)^{\otimes s}$. A typical element is written $\left[\alpha_{1}|\cdots| \alpha_{s}\right]$ and has bidegree $(s, t)$, where $t=\sum_{i=1}^{s} \operatorname{deg} \alpha_{i}$ is the internal degree inherited from $A$. Let $C^{s, t}$ be spanned by elements of bidegree $(s, t)$. There is a differential $\delta: C^{s, t}(A) \longrightarrow C^{s+1, t}(A)$, which may be computed as follows. Let $\mu^{*}$ be the composite $A^{*} \stackrel{\mu_{A}^{*}}{\longrightarrow}(A \otimes A)^{*} \stackrel{\theta^{-1}}{\longrightarrow} A^{*} \otimes A^{*}$, where $\mu_{A}^{*}$ is dual to the multiplication map $\mu_{A}$ of $A$, and $\theta$ is the isomorphism defined more generally by

$$
\theta: M^{*} \otimes N^{*} \longrightarrow(M \otimes N)^{*}, \quad \theta(f \otimes g)(m \otimes n)=(-1)^{\operatorname{deg} g \operatorname{deg} m} f(m) g(n)
$$

for $A$-modules $M$ and $N$. If $\mu^{*}(\alpha)=\sum_{r} \alpha_{r}^{\prime} \otimes \alpha_{r}^{\prime \prime}$, then $\delta$ is given by

$$
\delta\left(\left[\alpha_{1}|\cdots| \alpha_{s}\right]\right)=-\sum_{1 \leq i \leq s ; r}(-1)^{e_{i, r}}\left[\alpha_{1}|\cdots| \alpha_{i, r}^{\prime}\left|\alpha_{i, r}^{\prime \prime}\right| \cdots \mid \alpha_{s}\right]
$$

where $e_{i, r}$ is the total degree of $\left[\alpha_{1}|\cdots| \alpha_{i, r}^{\prime}\right]$. The cohomology groups of $A$ are then $H^{s, t}(A)=H^{s}\left(C^{*, t}(A), \delta\right)$.

$C^{*}(A)$ has the structure of a differential graded algebra with product

$$
\left[\alpha_{1}|\cdots| \alpha_{s}\right] \cup\left[\beta_{1}|\cdots| \beta_{s^{\prime}}\right]=\left[\alpha_{1}|\cdots| \alpha_{s}\left|\beta_{1}\right| \cdots \mid \beta_{s^{\prime}}\right] .
$$

The induced product in cohomology coincides with the Yoneda Ext product as given in [1, 2.2].

A.2. Massey products. The following material on Massey products can be found in [16. A1.4] and 8]. We recall the definition of the 3-fold Massey product. Let $(U, \delta)$ be a differential bigraded algebra. Let $\alpha_{i} \in H^{s_{i}, t_{i}}(U)$ be represented by $a_{i}$, and assume $\alpha_{1} \alpha_{2}=\alpha_{2} \alpha_{3}=0$. Let $s=s_{1}+s_{2}+s_{3}, t=t_{1}+t_{2}+t_{3}$. For any element $a \in U$, let $\bar{a}=(-1)^{\operatorname{deg}(a)+1} a$ where, as usual, degree means total degree. Let $\delta\left(u_{i}\right)=\bar{a}_{i} a_{i+1}$. Then the Massey product $\left\langle\alpha_{1}, \alpha_{2}, \alpha_{3}\right\rangle \in H^{s-1, t}(U)$ is the class represented by the cocycle $\bar{a}_{1} u_{2}+\bar{u}_{1} a_{3}$. This 3 -fold product is not well defined because the choices made in its construction are not unique. The choices of $a_{i}$ do not matter, but the $u_{i}$ could each be altered by adding a cocycle $x_{i} \in U^{s_{i}+s_{i+1}-1, t_{i}+t_{i+1}}$. This would alter $\left\langle\alpha_{1}, \alpha_{2}, \alpha_{3}\right\rangle$ by an element of the form $\alpha_{1} \llbracket x_{2} \rrbracket+\llbracket x_{1} \rrbracket \alpha_{3} \in \alpha_{1} H^{s_{2}+s_{3}-1, t_{2}+t_{3}}(U)+H^{s_{1}+s_{2}-1, t_{1}+t_{2}}(U) \alpha_{3}=\operatorname{Ind}\left\langle\alpha_{1}, \alpha_{2}, \alpha_{3}\right\rangle$. By $\llbracket x \rrbracket$ we mean the class of $x$ in $H^{*}(U)$. The group $\operatorname{Ind}\left\langle\alpha_{1}, \alpha_{2}, \alpha_{3}\right\rangle$ is called the indeterminacy of $\left\langle\alpha_{1}, \alpha_{2}, \alpha_{3}\right\rangle$. If the indeterminacy is trivial, then $\left\langle\alpha_{1}, \alpha_{2}, \alpha_{3}\right\rangle$ is a single class in $H^{*} *(U)$. 
Definition A.2. With notation as above, $\left\langle\alpha_{1}, \alpha_{2}, \alpha_{3}\right\rangle \subset H^{s-1, t}(U)$ is the coset of $\operatorname{Ind}\left\langle\alpha_{1}, \alpha_{2}, \alpha_{3}\right\rangle$ represented by $\bar{a}_{1} u_{2}+\bar{u}_{1} a_{3}$.

Similarly, one can define $k$-fold Massey products $\left\langle\alpha_{1}, \ldots, \alpha_{k}\right\rangle$ for $k>3$. Let $\alpha_{i} \in H^{s_{i}, t_{i}}$ be represented by $a_{i}$, and let $s_{i, j}=\sum_{r=i+1}^{j}\left(s_{r}-1\right), t_{i, j}=\sum_{r=i+1}^{j} t_{r}$, $s=s_{1, k}+2$, and $t=t_{1, k}$.

Definition A.3. A collection of cochains $\mathcal{A}=\left\{a_{i, j} \in U^{s_{i, j}+1, t_{i, j}}\right\}_{0 \leq i<j \leq k}$ is said to be a defining system for the $k$-fold Massey product $\left\langle\alpha_{1}, \ldots, \alpha_{k}\right\rangle$ if

$$
\begin{aligned}
a_{i-1, i} & =a_{i} \text { for } i=1, \ldots, k, \\
\delta a_{i, j} & =\sum_{i<r<j} \bar{a}_{i, r} a_{r, j} .
\end{aligned}
$$

The cocycle $c(\mathcal{A}) \in U^{s, t}$ defined by

$$
c(\mathcal{A})=\sum_{r=1}^{k-1} \bar{a}_{1, r} a_{r, k}
$$

is called the related cocycle of $\mathcal{A}$. The $k$-fold Massey product $\left\langle\alpha_{1}, \ldots, \alpha_{k}\right\rangle \subset$ $H^{s, t}(U)$ consists of all classes $\llbracket c(\mathcal{A}) \rrbracket$, where $\mathcal{A}$ ranges over all defining systems of $\left\langle\alpha_{1}, \ldots, \alpha_{k}\right\rangle$.

It can be shown that the set $\left\langle\alpha_{1}, \ldots, \alpha_{k}\right\rangle$ does not depend on the choice of representatives of the $\alpha_{i}$. The $k$-fold product is then a cohomology operation of $k$ variables that is defined if all the lower products $\left\langle\alpha_{i}, \ldots, \alpha_{j}\right\rangle$ for $0<j-i<k-1$ are defined and contain zero. Here the double product $\left\langle\alpha_{i}, \alpha_{i+1}\right\rangle$ is understood to be the regular product $\alpha_{i} \alpha_{i+1}$. The indeterminacy can get quite complicated, however [16, A1.4.4]. Kraines has constructed a "restricted" $k$-fold Massey product on the cohomology of topological spaces which has no indeterminacy [8, Section 3]. This construction can be carried over to the Hopf algebra setting [7 Section 3]. If $\alpha_{i}=\alpha$ for all $i$ and $\alpha^{2}=0$, one can define the symmetric Massey product $\langle\alpha\rangle^{k}$.

Definition A.4. Let $\alpha \in H^{s, t}(U)$ be a class such that $\alpha^{2}=0$. A collection of cocycles $\mathcal{A}=\left\{a_{i} \in U^{i(s-1)+1, i t}\right\}_{i=1}^{k-1}$ is called a defining system for the symmetric Massey product $\langle\alpha\rangle^{k}$ if

$$
\begin{aligned}
& a_{1} \text { represents } \alpha, \\
& \delta a_{i}=\sum_{r=1}^{i-1} \bar{a}_{r} a_{i-r} \text { for } 2 \leq i \leq r-1 .
\end{aligned}
$$

The cocycle $c(\mathcal{A}) \in U^{k(s-1)+2, k t}$ defined by

$$
c(\mathcal{A})=\sum_{r=1}^{k-1} \bar{a}_{r} a_{k-r} \in U^{k(s-1)+2, k t}
$$

is called the related cocycle of $\mathcal{A}$. The symmetric product $\langle\alpha\rangle^{k}$ is then the set of all classes $u \in H^{k(s-1)+2, k t}(U)$ which are represented by some $c(\mathcal{A})$, where $\mathcal{A}$ is a defining system for $\langle\alpha\rangle^{k}$. If $\langle\alpha\rangle^{k}$ is defined and equal to a single homology class then $\langle\alpha\rangle^{k}$ is said to be defined with zero indeterminacy. 
Lemma A.5. Let $p$ be odd, and let $A$ be a Hopf algebra over $\mathbb{F}_{p}$.

(1) If $\alpha \in H^{2 s+1, t}(A)$, then $\langle\alpha\rangle^{p}$ is defined with zero indeterminacy 7, Lemma 14].

(2) If $\alpha \in H^{1, t}(A)$, then $\langle\alpha\rangle^{p}=-\widetilde{\beta \mathcal{P}}^{0}(x)$ [11, Remark 11.11].

\section{A.3. Calculations. Let}

$$
\begin{array}{ll}
A=\mathbb{F}_{p}[x] /\left(x^{p^{n}}\right), & \operatorname{deg} x=m \text { even, } n \geq 1, \quad p \text { odd }, \\
A=\mathbb{F}_{2}[x] /\left(x^{2^{n}}\right), \quad \operatorname{deg} x=m, n>1, \quad p=2 .
\end{array}
$$

The augmentation in both cases is defined by $\epsilon(x)=0$. It is known that the cohomology of such a truncated polynomial algebra is the tensor product of an exterior algebra on a one-dimensional class and a polynomial algebra on a two dimensional class. We would like to identify specific generators in the cobar complex $C^{*}(A)=\operatorname{Tens}\left(I(A)^{*}\right)$.

Choose for $I(A)$ the natural basis $\left\{x^{i}, 0<i<p^{n}\right\}$. Let $\left\{y_{i}\right\}$ be the dual basis for $I^{*}$. Then

$$
\delta\left[y_{k}\right]=\sum_{\substack{i+j=k \\ 0<i<k}}\left[y_{i} \mid y_{j}\right] .
$$

Clearly, in $C^{1,2 m}(A), z=\left[y_{1}\right]$ is a cocycle. Let $e=\sum_{i=1}^{p^{n}-1}\left[y_{i} \mid y_{p^{n}-i}\right]$. A routine calculation shows that $e$ is a nonbounding cocycle. By abuse of notation, we can thus take $z$ and $e$ to be the generators of $H^{*}(A)$.

Lemma A.8. Let $A$ be a truncated polynomial algebra as in (A.6). Then $H^{*}(A) \simeq$ $\Lambda(z) \otimes \mathbb{F}_{p}[e]$, with $z \in H^{1, m}, e \in H^{2, m p^{n}}$ as defined above. Moreover, the symmetric Massey product $\langle z\rangle^{p^{n}}=e$ with zero indeterminacy.

Proof. Choosing $a_{i}=\left[y_{i}\right]$ gives a defining system for $\langle z\rangle^{p^{n}}$. Its related cocycle is $\sum_{r=1}^{p^{n}-1}\left[y_{r} \mid y_{p^{n}-r}\right]$, which represents $e$. There is no indeterminacy because for each $i$, there is no other choice of $a_{i}$.

Remark. If $p=2, n=1$, then $A=\mathbb{F}_{2}[x] /\left(x^{2}\right) \simeq \Lambda[x]$, and $H^{*}(A) \simeq \mathbb{F}_{2}[z]$ with $z=\left[x^{*}\right] \in H^{1, \operatorname{deg} x}$.

Summarizing,

Theorem A.9. Let $A=\mathbb{F}_{p}[x] /\left(x^{p^{n}}\right)$, then the following Steenrod operations and symmetric Massey products are present in $H^{*}(A)$ :

(1) $\langle z\rangle^{p^{n}}=e, \widetilde{\mathcal{P}}^{1} e=e^{p}$ for $p$ odd,

(2) $\widetilde{\beta \mathcal{P}}^{0} z=-e$ for $p$ odd, $n=1$,

(3) $\langle z\rangle^{2^{n}}=e, \widetilde{S q}^{2}(e)=e^{2}$ for $p=2, n \geq 2$,

(4) $\widetilde{S q}^{1}(z)=z^{2}$ for $p=2, n=1$.

Proof. These are consequences of the axioms in Theorem 2.2 and of the results just stated. 


\section{REFERENCES}

1. J. F. Adams, On the non-existence of elements of Hopf invariant one, Ann. of Math. (2) 72 (1960), 20-104. MR 25:4530

2. Alexander Beilinson, Victor Ginzburg, and Wolfgang Soergel, Koszul duality patterns in representation theory, J. Amer. Math. Soc. 9 (1996), no. 2, 473-527. MR 96k:17010

3. Norman Blackburn, Generalizations of certain elementary theorems on p-groups, Proc. London Math. Soc. (3) 11 (1961), 1-22. MR 23:A208

4. Jill Dietz, Stable splittings of classifying spaces of metacyclic p-groups, $p$ odd, J. Pure Appl. Algebra 90 (1993), no. 2, 115-136. MR 95f:55014

5. Bertram Huppert and Norman Blackburn, Finite groups. II, Springer-Verlag, Berlin, 1982. MR 84i:20001a

6. Daniel S. Kahn, cup - $i$ products and the Adams spectral sequence, Topology 9 (1970), 1-9. MR 40:6552

7. Stanley O. Kochman, Symmetric Massey products and a Hirsch formula in homology, Trans. Amer. Math. Soc. 163 (1972), 245-260. MR 48:9721

8. David Kraines, Massey higher products, Trans. Amer. Math. Soc. 124 (1966), 431-449. MR 34:2010

9. Michel Lazard, Sur les groupes nilpotents et les anneaux de Lie, Ann. Sci. Ecole Norm. Sup. (3) 71 (1954), 101-190. MR 19:529b

10. Clas Löfwall, On the subalgebra generated by the one-dimensional elements in the Yoneda Ext-algebra, Algebra, algebraic topology and their interactions (Stockholm, 1983), Lecture Notes in Math., vol. 1183, Springer, Berlin, 1986, pp. 291-338. MR 88f:16030

11. J. Peter May, A general algebraic approach to Steenrod operations, The Steenrod Algebra and its Applications (Proc. Conf. to Celebrate N. E. Steenrod's Sixtieth Birthday, Battelle Memorial Inst., Columbus, Ohio, 1970), Springer, Berlin, 1970, pp. 153-231. MR 43:6915

12. John W. Milnor and John C. Moore, On the structure of Hopf algebras, Ann. of Math. (2) 81 (1965), 211-264. MR 30:4259

13. Stewart B. Priddy, Koszul resolutions, Trans. Amer. Math. Soc. 152 (1970), 39-60. MR 42:346

14. _ Primary cohomology operations for simplicial Lie algebras, Illinois J. Math. 14 (1970), 585-612. MR 42:5253

15. Daniel G. Quillen, On the associated graded ring of a group ring, J. Algebra 10 (1968), 411-418. MR 38:245

16. Douglas C. Ravenel, Complex cobordism and stable homotopy groups of spheres, Academic Press Inc., Orlando, FL, 1986. MR 87j:55003

17. Clarence Wilkerson, The cohomology algebras of finite-dimensional Hopf algebras, Trans. Amer. Math. Soc. 264 (1981), no. 1, 137-150. MR 82e:16019

Department of Mathematics, Whittier College, Whittier, California 90608 E-mail address: jmauger@whittier.edu

Current address: Department of Mathematics and Computer Science, California State University, Channel Islands, Camarillo, California 93012

E-mail address: justin.mauger@csuci.edu 